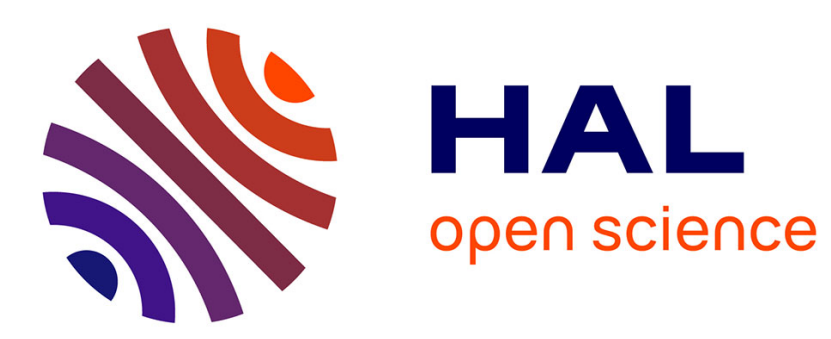

\title{
Accurate post-mortem alignment for Focused Ion Beam and Scanning Electron Microscopy (FIB-SEM) tomography
}

\author{
Hui Yuan, B van de Moortele, Thierry Epicier
}

\section{- To cite this version:}

Hui Yuan, B van de Moortele, Thierry Epicier. Accurate post-mortem alignment for Focused Ion Beam and Scanning Electron Microscopy (FIB-SEM) tomography. 2020. hal-03024445

\section{HAL Id: hal-03024445 \\ https://hal.science/hal-03024445}

Preprint submitted on 25 Nov 2020

HAL is a multi-disciplinary open access archive for the deposit and dissemination of scientific research documents, whether they are published or not. The documents may come from teaching and research institutions in France or abroad, or from public or private research centers.
L'archive ouverte pluridisciplinaire HAL, est destinée au dépôt et à la diffusion de documents scientifiques de niveau recherche, publiés ou non, émanant des établissements d'enseignement et de recherche français ou étrangers, des laboratoires publics ou privés. 


\title{
Accurate post-mortem alignment for Focused Ion Beam and Scanning Electron Microscopy (FIB-SEM) tomography
}

\author{
H. Yuan ${ }^{1,2 *}$, B. Van de Moortele ${ }^{2}$, T. Epicier ${ }^{1,3} \S$
}

1. Université de Lyon, INSA-Lyon, Université Claude Bernard Lyon1, MATEIS, umr CNRS 5510, 69621 Villeurbanne Cedex, France

2. Université de Lyon, ENS-Lyon, LGLTPE, umr CNRS 5276, 69364 Lyon 07, France 3. Université de Lyon, Université Claude Bernard Lyon1, IRCELYON, umr CNRS 5256, 69626 Villeurbanne Cedex, France

\begin{abstract}
Drifts in the three directions (X, Y, Z) during the FIB-SEM slice-and-view tomography is an important issue in 3D-FIB experiments which may induce significant inaccuracies in the subsequent volume reconstruction and further quantification of morphological volume parameters of the sample microstructure. Cross-correlation is frequently applied directly to the cross-section image series for aligning FIB sliced images. This solution is hazardous and can be flawed as it has been easily demonstrated by a dedicated test experiment. As a result, a novel aligning procedure based on the quantification of the topography of the sample surface has been developed. This new approach will be compared to the common cross-correlation methods, as well as another approach consisting in using an artificial reference marker fabricated during the FIB procedure. All these methods will then be discussed in terms of accuracy and liability.
\end{abstract}

Key words: FIB-SEM tomography, drifts, post-mortem alignment, reference markers, crosscorrelation, surface topography

* Corresponding Author:

Hui Yuan, Canadian Centre for Electron Microscopy, McMaster University, Hamilton, ON L8S 4M1, Canada. Tel: +1 9055259140 Ext. 24071; email: yuanh9@mcmaster.ca.

§Present address: Thierry Epicier, Université de Lyon, Université Claude Bernard Lyon1, IRCELYON, umr CNRS 5256, 69626 Villeurbanne Cedex, France; email : thierry.epicier@ireclyon.univ-lyon1.fr 


\section{Highlights:}

- The limitations and potential dangers of the current cross-correlation alignment method for FIB-SEM tomography are demonstrated when undesirable (X, Y, Z) drifts occur during the acquisition of the $3 \mathrm{D}$ image series.

- Proper references of alignment, including the quantification of the surface topography of the sample, and the use of a FIB drilled hole, have therefore been developed and compared.

- The topography based on the sample surface leads to the more accurate alignment.

- The measurement of layer thickness in the FIB slicing direction $\mathrm{z}$, an important parameter in FIB-SEM tomography is also discussed and well evaluated by the surface topography method. 


\section{Introduction}

\subsection{FIB-SEM tomography}

The slice-and-view tomography by Focused Ion Beam - Scanning Electron Microscopy (FIBSEM tomography, or 3D FIB-SEM) in a dual-beam (DB) FIB instrument has currently become a powerful approach to acquire real three-dimensional (3D) information of a moderate analysed volume $\left(10 \sim 1000 \mathrm{\mu m}^{3}\right)$ from a given material at a resolution of a few nanometres. This scale bridges the gap between other microscopy tomography techniques. X-ray tomography offers currently at a resolution of a fraction of micrometre [1-3]; Transmission Electron Microscopy (TEM) tilting nano-tomography gives typically access to one nanometre [4,5] and even atomic resolution in favourable cases [6-8], as does Atom Probe Tomography (APT) [9,10]. This technique has thus largely been applied in various domains, such as materials science [11-13], geological science $[14,15]$ and biology science $[16,17]$. However, high-resolution 3D techniques like TEM tilting APT are limited to very small analysed volumes, and they generally require a long and accurate sample preparation. Thus, they are not always perfectly adapted to the problem of interest, especially in terms of size of the analysed volume and the relevant scale at which its heterogeneous microstructure needs to be quantified. In this respect, 3D FIB-SEM is a very good compromise between resolution and volume size. Especially with the recent application of Xe plasma ion beam[18], broad $\mathrm{Ar}^{+}$beam [19], and Femtosecond laser [20], the analysing volumes can be increased largely.

FIB-SEM tomography (Figure 1) is generally a two-step procedure consisting of 1) data acquisition and 2) post-mortem data treatment.

The acquisition step starts with the preparation of the working area (typically in ' $U$ ' shape), including milling a flat surface (XY plane represented by the AA' segment in projection, see Figure 1) and associated slices, especially in front of this surface to avoid any shadowing effect during SEM observation. Then, the cross-sectional surface is successively imaged and sliced by removing continuously a thin layer of nominal constant thickness in the $\mathrm{Z}$ direction, thanks to ion beam machining. More detailed experimental descriptions can be found elsewhere [11,12]. Additionally, Electron Backscatter Diffraction (EBSD) [21-23] or Energy-Dispersive X-ray spectroscopy (EDX) [24-26] experiments can be simultaneously carried out in order to collect complementary crystallographic or chemical information, either separately or at the same time [27]. The post-mortem treatment concerns alignment, filtering, reconstruction and visualisation of collected data; these treatments constitute an important process to produce interpretable, quantitative and reliable 3D results. On the contrary of other microscopy-based tomographic acquisitions such as of tilt series in electron microscopy or X-ray tomography, or of evaporation ion bursts APT, the FIB-SEM reconstruction of the analysed volume should be straightforward without needing any restricting assumptions or dedicated algorithms. In FIB-SEM, the volume is expected to be ideally sliced plane-by-plane, each slice having a priori a constant thickness. In practice, problems arise from various sources leading to possible misalignments of the image series in the $\mathrm{X}, \mathrm{Y}$ and $\mathrm{Z}$ directions. According to the nature of the sample, the ion and electron beams 
energy to respectively machine and image the surface, the size of recorded images and the quantity of matter scanned in depth, the acquisition of the tomographic series may take hours or even days [28]. Consequently, drifts of the region of interest (ROI) appear to be essentially unavoidable. These drifts will be described in more details in the next sub-sections, which also intend to hierarchize the procedures to account, limit and compensate them either by on-line or post-mortem corrections.

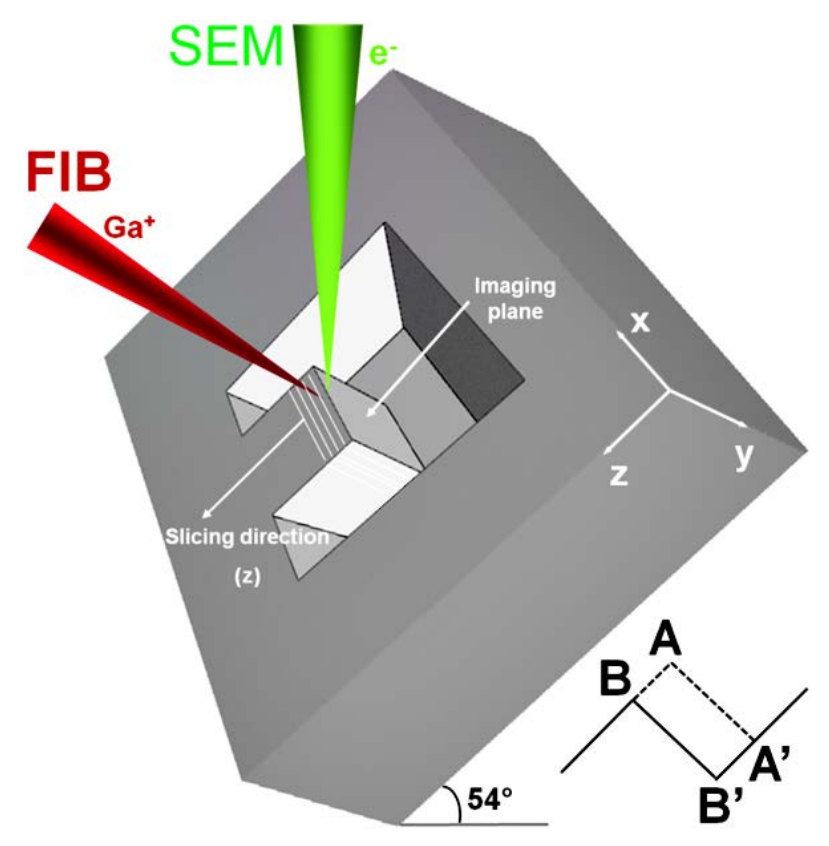

Figure 1. Sample geometry during a FIB-SEM tomography sequence with the Carl Zeiss Nvision 40 FIB/SEM crossbeam ${ }^{\circledR}$ (adapted from [11]). The sketch in the lower right corner schema shows the projection of imaged surface in the plane YZ) during one imaging-sectioning cycle: the electron beam is scanned on the plane AA' to produce a first image. The volume of interest (VOI) of the material defined by the rectangle ABB' $A^{\prime}$ is eroded by the ion beam then a second image of the plane BB' is captured after having adapted the working distance.

\subsection{Drift problems during image acquisition and correction methods}

As mentioned above, spatial drifts occur because of unavoidable displacements of the ROI during the acquisition step. They may also be amplified by various reasons from different origins, such as: charging or electrostatic effects for non-conductive materials, contraction or deformation of sensitive samples (like polymer or biologic matter), or mechanical or electromagnetic instabilities caused by external perturbations or insufficiently corrected stray fields. Along the advancement of milling in Z-direction, consequences are that: i) the SEM focus may be lost during the acquisition (a major limitation if the procedure is run automatically without any adequate automatic correction). ii) the thickness of removed matter during slice milling becomes irregular, 
which also degrades the quality of the reconstruction. And drifts along $\mathrm{X}$ and $\mathrm{Y}$ directions makes the actual ROI may be cropped to a very small area after a final, e.g., post mortem alignment.

When some studies have focused on the improvement of the environment around the microscope, or hardware and software solutions to stabilize the working conditions of the instrument itself (see for example [29]), most of corrections consists in registration methods applied to the shifted images, either during the acquisition or post-mortem on the acquired image stack.

The first correction strategy consists in trying to compensate drifts on-line during the acquisition of images [11,25,30-32]. A systematic correction can be made regularly every $n$ slices (the faster the drift, the smaller the value of $n$ ): a pattern recognition algorithm can then be used to realign the successive slices by moving the sample or adapting the position of the ROI accordingly. Regarding (X, Y) drifts, the region of interest (ROI) should remain centred to avoid significant cropping after the final post mortem alignment of images greatly misaligned. At this stage, it is then only question of keeping the ROI in the field of view at the chosen magnification. This is a particularly critical point when the sample is rotated during the slice-and-view process as it is performed to mix the tomography approach with a crystallographic analysis by Electron Backscatter Electron Diffraction (EBSD) as performed by Zaefferer et al. [33]. Drift corrections can be run using a cross-correlation based alignment of a fixed fiducial marker milled on the top surface [32] as proposed in Atlas software developed by Fibics company for Zeiss FIB/SEM instruments or of part of the ROI itself. As a typical example a dynamic procedure for live shift correction was developed [30] on a Carl Zeiss instrument by using the manufacturer API (application programming interface) and the 'MultiStackReg' plugin [31] in the Fiji [32] open source software.

Such corrections using either the ROI microstructure or artificial markers as a reference essentially rely on cross-correlation techniques, which are also the most common basis of post mortem alignments, a second and complementary strategy to correct ideally any residual drift remaining after the acquisition [11,32,34,35]; they will thus be discussed in the next section. As for $\mathrm{Z}$ drift, an ideally constant slicing layer thickness is expected by cross-correlating a fixed reference marker on the top surface and adjusting beam shifts. Such on-line corrections have nevertheless drawbacks: they lead to an increase of the total image acquisition time; additional ion beam instability can occur because of switching the aperture between high milling beam current and low imaging current; and the milling steps with an intense ion beam current can destroy the protection layer then even the fiducial makers.

It is important to note that this final post-processing alignment can only correct residual displacements in the (X, Y) planes since the Z-slicing has been destructive. Consequently, crossing diagonal $[11,36]$ markers on the $\mathrm{X}-\mathrm{Z}$ top surface of the sample are generally machined in order to estimate post mortem the thickness of each slice before the reconstruction. This has been adapted as an automatic feature in Atlas software (Fibics Inc., Ottawa, Canada; www.fibics.com) for FIB/SEM tomography running in Zeiss instruments, Jones et al. [37] milled crossed fiducial lines on multiple deposited layers to improve the accuracy of this thickness measurement. Moreover, 
Mangipudi et al. [38] prepared a wedge-shaped geometry to calculate the thickness from the evolution of the ROI width in the successive cross-sectional images.

\subsection{Post mortem alignment procedures and their limitations}

A post mortem alignment is indeed required even if no obvious external source has produced severe drifts, and even if on-line corrections have been run: in general, drifts may occur at a spatial scale making it very delicate to run efficient and fast on-line corrections. post-mortem (X, Y) alignment usually consists in operating translations by applying cross-correlation-based operations to successive images. Several softwares can be used, like Amira ${ }^{\circledR}$ (Visualization Sciences Group, FEI, Burlington, MA, USA), Avizo ${ }^{\circledR}$ (Visualization Sciences Group, FEI, Burlington, MA, USA), Aphelion $^{\circledR}$ (ADCIS, Saint-Contest, France), IDL ${ }^{\circledR}$ (Exelis, McLean, VA, USA), IMOD [39], plugin 'StackReg' [40] or 'MultiStackReg' in Fiji package. To do so, several approaches have been utilised. Some authors $[24,41,42]$ use the interface between the X-Y surface of the sample and the $\mathrm{X}-\mathrm{Z}$ top protected layer as an alignment reference for cross-correlation. Further refinements employ fiducial markers prepared on the $X-Z$ top surface (usually the deposited protection layer) and parallel to the $\mathrm{Z}$ direction: for example, a set of drilled positional markers [41-43], or linear markers [24,25,44]. Alternatively, markers (usually crosses and circles) can also be positioned far from the milling area and then used for alignment at lower magnification than the working magnification [25], or also at the same magnification [38] by sacrificing the effective SEM imaging area. Otherwise, FIB milled micron-size holes on the imaging surface are proposed as marker [45].

In practice, the post-mortem alignment methods described above may be efficient, but they can indeed be quite inaccurate for several reasons listed above and linked to the fact that the exact geometry of the sample is, essentially, unknown:

(i) Any cross-correlation routine will align features which 'in reality' should not be aligned: this can indeed create flawed microstructure with perfect alignments from a numerical point of view but not representative from a structural point of view. An illustration is given in Figure

2 , where we can imagine a prominent unaligned feature of the real microstructure that will give rise to a perfect but false alignment. We will see later on practical examples that significant misalignments are easily produced by cross-correlation, although the 3D microstructure seems to be perfectly aligned by eye inspection.

(ii) During milling, markers may be partially covered by undesirable re-deposition, and then they can lose their efficiency. Some sophisticated method can be used to prevent to 'erase' the alignment markers: for example, a dedicated alignment marker geometry has been proposed [46] using an elaborated scheme of milling and deposition of TEOS / Pt TEOS, in order to conserve a good SEM contrast and avoid any visibility loss due to contamination by FIB redeposition. This approach does not apply in the case of a non-flat or inclined surface as 
discussed in (iii) and does not permit a further estimation of eroded layer thickness for the acquisition.

(iii) Alignment based on the top surface (or the top line intersection of the XY and XZ planes) may be wrong if this surface is not flat and/or inclined - not parallel to the microscope stage -).

b)

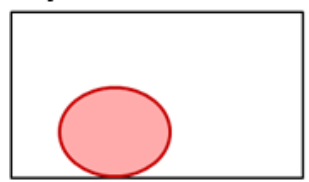

c)
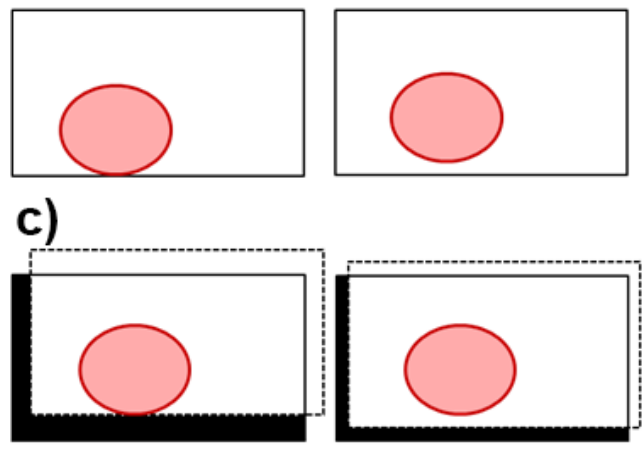

a)
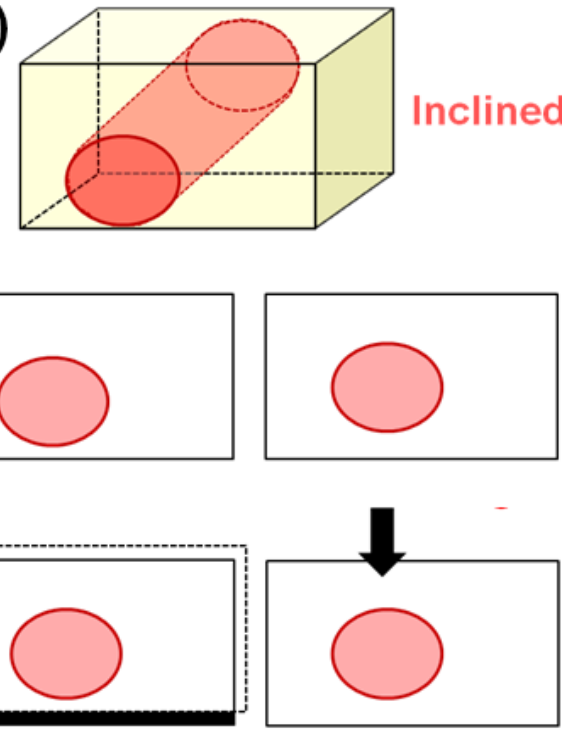
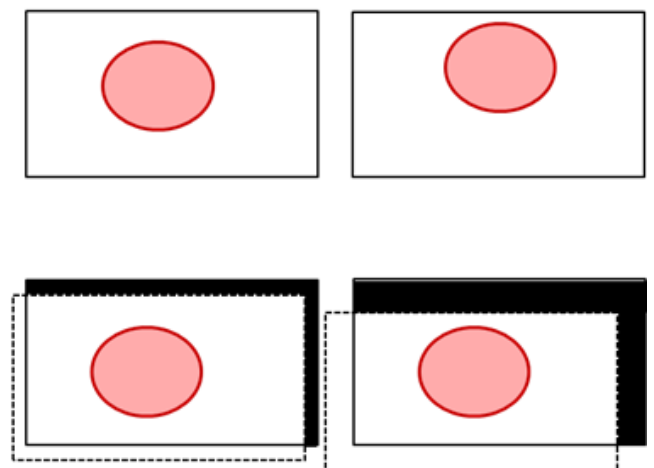

Figure 2. Illustration of a wrong alignment produced by a cross-correlation approach in the case of an inclined (according to horizontal plane) object in the VOI (a): for clarity, this 'object' is considered to be a cylindrical hole, of inclusion in the material. b): Expected successive sections in the ideal case where no re-alignment is required. c): result of a cross-correlation alignment of the successive elliptical sections with respect to the reference image (the central image arrowed), thus creating a wrong microstructure.

According to the above points, the present work will first report a dedicated test experiment demonstrating the difficulties in the alignment procedure based on cross-correlation calculations.

Secondly, we will propose two other types of references for the post-mortem alignment of FIBSEM tomography image series: one based on the topography of the sample surface and one using a reference FIB- drilled tubular hole crossing the ROI. The comparison of these alignment results with that from common alignment routines show that the method using the top surface topography leads to a better accuracy. Furthermore, its ability to provide a better estimate of the averaged eroded slice thickness will be discussed.

\section{Materials and methods}

The sample used here is a non-conductive dehydrated serpentine. This mineral is present in abundance in the earth mantle and is extensively studied especially by high temperature and high pressure deformation to simulate the underground environment and furthermore study earthquakes $[47,48]$. Serpentine exhibits interesting microstructural features (porosity, fracture, chemical heterogeneities) and is a good candidate for the present FIB-SEM tomography study. 
This work is performed in a Carl Zeiss Nvision 40 crossbeam ${ }^{\circledR}$ FIB station (Carl Zeiss AG, Oberkochen, Germany). It is composed of an inclined SII ion optic column (Seiko Instruments Inc., Tokyo, Japan) with Gallium ion (Ga+) liquid metal ion source (LMIS) and a vertical Zeiss Gemini $^{\circledR}$ electron column of field emission gun (FEG). Two micro-manipulators (Klocke Nanotechnik, Aachen, Germany) are also equipped. The sample geometry during the experimental acquisition of tomography is illustrated in Figure 1.

\section{Issue of commonly used methods to align image series in FIB-SEM tomography}

To perform a complete analysis of the accuracy of cross-correlation based alignment approaches, the geometry of the sample must be properly controlled. For this reason, a small volume was completely extracted from the bulk sample in order to access easily to lateral surfaces, where specific markers were FIB-prepared. Extracting a micro-volume from a bulk sample was a long and tedious operation, but it offered the advantage to suppress any shadowing, and to minimize any possible re-deposition of sputtered material. Figure 3a shows the $20 \times 40 \times 16 \mu^{3}$ micro-volume that has been extracted from the bulk sample by the classical 'lift-out' method $[49,50]$. Once extracted, the small volume was glued on a standard copper support for TEM lamellae (Figure 3b-c). 4 linear markers were milled (Figure 3d) on the lateral side with a low ionic current, and further filled with deposited tungsten to provide a good contrast of these trenches when viewed edge-on during the slice-and-view acquisition. The two parallel lower lines were intended to allow a good stack alignment along the $\mathrm{Y}$ direction, and the two intersecting upper lines allowed the thickness increment to be checked in the $\mathrm{Z}$ direction. As shown in the front image of Figure 4, the trace of these markers on the lateral side should also allow the alignment in the $\mathrm{X}$ direction.

Then, a tomographic series of images was acquired according to the experimental parameters listed in table 1. After the experiment, different cross-correlation based alignments were applied on several reference areas: 1) the central area of ROI, 2) the left border of ROI with fiducial markers, and 3-4) large and small areas respectively including the interface between the topdeposited carbon layer and the ROI. In parallel, a 'correct' alignment was performed by aligning manually the markers shown in Figure 3d.

Table 1. Experimental conditions for a study case in order to test the accuracy of alignment procedures based on cross-correlation

\begin{tabular}{llll}
\hline FIB & & SEM & \\
\hline Step in Z & $25 \mathrm{~nm}$ & SEM pixel size & $12.5 \mathrm{~nm}$ \\
Milling probe & $700 \mathrm{pA}$ & Aperture size & $60 \mu \mathrm{m}$ \\
Milling depth & $15 \mu \mathrm{m}$ & Detectors & Inlens, EsB \\
FIB EHT & $30 \mathrm{kV}$ & SEM EHT & $1.5 \mathrm{kV}$ \\
\hline
\end{tabular}



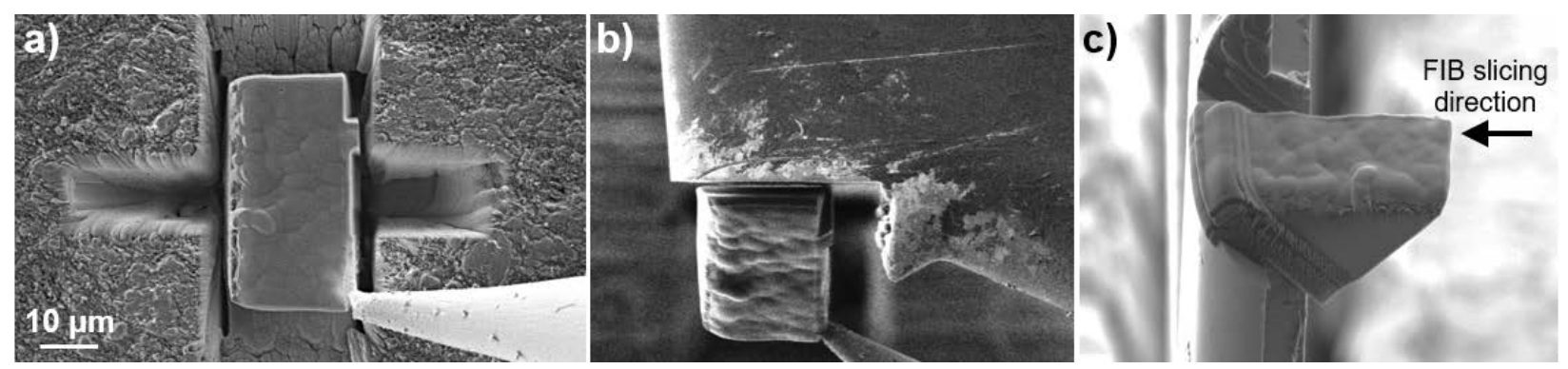

Figure 3. a): SEM view of the micro-volume extracted from a bulk sample (serpentine) just before 'liftout' with the help of a micro-manipulator. b): Montage of the micro-volume on an eroded Cu support. c): Wedge-shaped block, once glued on the Cu support before starting the slice-and-view procedure.

Results are shown in Figure 4 where the different alignments are illustrated by the side view of the surface where the specific markers were machined. Surprisingly, none of the reference areas gives a satisfactory result, although all aligned sequences were good-looking when checked in the Z-direction (see videos in Supplementary Information). The simplest reference area $\mathrm{n}^{\circ} 1$ leads to an alignment totally controlled by the isotropy of the microstructure. This procedure appears sufficient when applied on-line in order to keep the ROI [30] in the SEM field of view. However, the alignment results in fluctuations owing to specific features in the microstructure, although the eye inspection of the reconstructed volume does not reveal any discontinuity, and suggests on the contrary a well-aligned isotropic microstructure. All other reference areas, which include edges (intersection of surfaces) lead to more 'straight', but clearly flawed alignments. This simply attests that those edges are not perpendicular to the viewing $\mathrm{Z}$ direction, which produces the same effect as what was illustrated in Figure 2: inclined lines are just re-aligned by the cross-correlation.

At first, this experiment demonstrates unambiguously that cross-correlation based alignments are not accurate. It must be recalled that in all cases shown in Figure 4, the eye inspection of the aligned volumes along the $\mathrm{Z}$ direction suggests a good alignment.

It secondly demonstrates that arrays of parallel or intersecting lines on a side and flat surface can be efficient in order to produce a correct alignment. Unfortunately, the preparation process of such a 'lift-out' block is a long step which cannot be applied for routine work. An alternative method would be to prepare a conventional excavation, like in Figure 1, but enlarging the lateral trench (like again on the adjacent part of ROI in Figure 1), in order to realize FIB markers as seen in Figure 3d. Lateral or top markers are further limited in terms of accuracy: when seen in the (X, $\mathrm{Y}$ ) projection, they essentially provide a reasonable accuracy in one of the $\mathrm{X}$ or $\mathrm{Y}$ directions, but a poor one in the other. For example, the lateral markers in the reference area $n^{\circ} 2$ in Figure 4 should be adapted for the alignment along $\mathrm{Y}$ but not along $\mathrm{X}$ since the $\mathrm{X}$ direction corresponds to the small depth of milled lines (even refilled with tungsten deposition). In fact, such lateral markers are even not adapted in our case because the reference area 2 includes the bottom surface of the ROI, the position of which is evolving all along the slicing process, making the alignment incorrect. Such limitations have been systematically investigated in a previous work [36] by studying various markers with gradually varied depth prepared at the top surface of sample. 

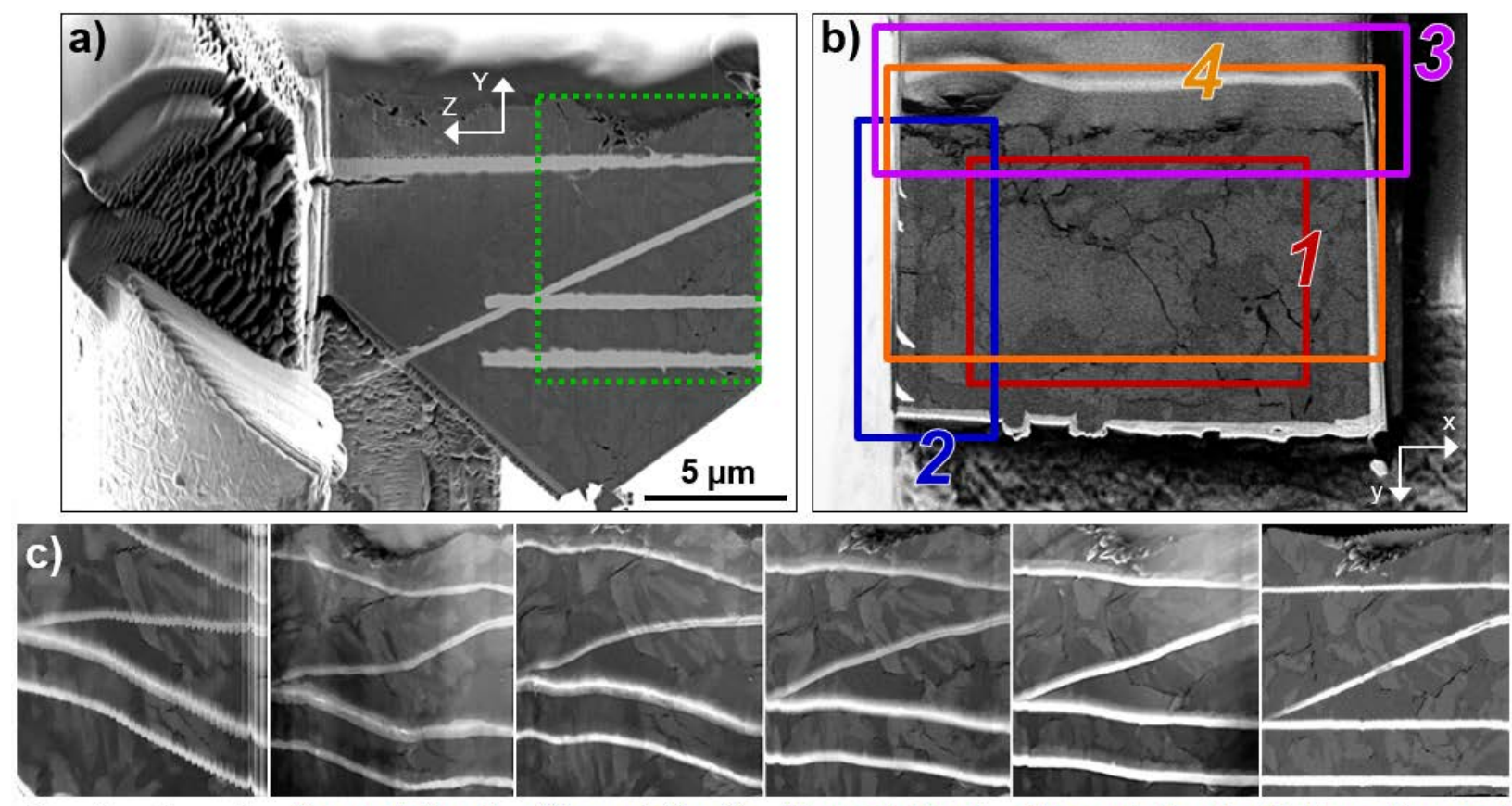

Raw (unaligned)

X-correlation 1

X-correlation 2

X-correlation 3

X-correlation 4 Markers alignment

Figure 4. Post-mortem alignment of a test experiment using an extracted micro-volume from a bulk serpentine sample. a): Side view of the sample similar for Figure 3c but once the lateral markers, i.e trenches milled and filled with tungsten, have been realized (after a brightness-contrast processing). b): Front (X, Y) view of the sliced area showing the different ROI used for alignment by cross (X)-correlation. c): Display of the side view (dotted frame in a) after alignment procedures as indicated (the contrast has been adapted independently according to the number of orthoslices averaged in each case). The first one is the unaligned data set which shows periodic discontinuities due to the application of live shift correction [30]. The last one was performed manually using the $(\mathrm{X}, \mathrm{Z})$ view.

According to these considerations, it must be concluded that cross-correlation alignments cannot be robust because the geometry of the sample is not 'robust': (i) the microstructure of the material is unknown by definition and cannot serve as a reference; (ii) the topography of any surface of the sample may influence the location, visibility of any marker and further induce inaccuracy because the true alignment of the microstructure does not correspond to a perfect alignment of those markers.

This last assessment is instructive and provides the basis of a possible 'robust' alignment: the topography of the top surface is inherent to the sample, and the reconstruction should reproduce it after a correct alignment. If we measure this topography a priori by an independent method, we can use it as a reference for the alignment. Because of the 3D nature of the top surface, he method should provide an alignment in the $3 \mathrm{X}, \mathrm{Y}, \mathrm{Z}$ directions at once. This is one of the strategies introduced in the following section. 


\section{Results of new tested alignment methods}

The conclusions of section 3 have led us to adopt another and new strategy for a more accurate and universal alignment. We need indeed a robust reference linked to the object itself. A first possibility is to use the surface of the sample, which can serve as a good reference if its topography can be reconstructed independently and without being affected during the 3D acquisition sequence. A second possible reference can be a significant feature intentionally produced by FIB machining throughout the volume to be sliced, like a hole drilled from one side to the other of the volume. These two new strategies will be developed in the following sub-sections.

\subsection{Sample surface topography}

\subsubsection{Reconstruction of the sample surface topography}

We already evoked that markers on the top surface can be good references for the alignment if one is certain that this surface has a known geometry, which means that it should essentially be flat. Different methods were employed to obtain such a nominally planar, flat sample surface: such as the ultramicrotome [51,52] and the vibratome [53] sections for biological sample, or polishing techniques for ceramic or metal based materials [54,55]. However, this preparation is not always possible, neither accurate for all samples. As a typical example, the deformed serpentine used previously (Figure 3) exhibits a lot of porosities or grooving effects inducing its brittleness and weak resistance to delamination during any preparation process, and it cannot be ascertained that the surface can be as flat as needed. Conversely, whatever the topography of the top surface, and especially if it is not perfectly flat, it can serve as an intrinsic reference for a subsequent alignment of the 3D stack of images produced during the FIB-SEM tomography experiment. To do so, we need to be able to characterize this surface in 3D before the slice-and-view sequence. This can be performed by stereophotogrammetry [56,57]. This stereoscopic approach consists in acquiring stereo-pairs or stereo-triplets of micrographs from which the surface reconstruction is deduced from simple trigonometric calculations retrieving the height of any detail at the surface from the differently inclined projections. Several in-house or commercial computer programs exist that have been widely used in the SEM community for various applications[58-67]. Figure 5 illustrates the measurement of the surface of our 'test' serpentine sample investigated by FIB-SEM tomography. We have acquired three images of the sample inclined at $-10^{\circ}, 0^{\circ}$ and $10^{\circ}$ as shown in Figure 5a to c. The magnification and scan conditions were adjusted to achieve a spatial resolution of $10 \mathrm{~nm} /$ pixel. We used the MEX software (Alicona SARL, Les Ulis, France) for the $3 \mathrm{D}$ reconstruction visualized in Figure 5d.

As stated above, this reconstructed surface will serve as a reference to align the stack of images produced during the 3D FIB-SEM experiment. Since these images are acquired along the Z direction, we need to produce line profiles, called 'MEX profiles' hereafter, of the reconstructed surface as $\mathrm{Y}(\mathrm{X})$ curves with increasing indexes for increasing $\mathrm{Z}$. An illustration of such a line is given in Figure 5d (corresponding to the top surface of a sectioned plane AA' in Figure 1). 


\subsubsection{Comparing top surface profiles obtained by FIB with MEX profiles from the reconstructed topography}

Before starting the FIB-SEM tomography acquisition, it appears necessary to properly protect the top surface of the sample to avoid any degradation during the FIB operation, which would invalidate any comparison of surface profiles obtained at each slicing step with the prior reconstructed surface. It was then covered by a thin layer of tungsten prior to the usual carbon deposition as a protective layer easily visible on the three micrograph reported on Figure 6. This layer also provides the advantage to produce a strong contrast in the SEM imaging, thus allowing an easy segmentation of the 2D line references on which the alignment will be based on. In addition, and again to compare all possible alignments strategies, we prepared a classic ' $U$ ' shape of the ROI, with a lateral (right) side widely enlarged in order to mill fiducial markers as performed in the lift-out block discussed above. These markers are seen edge-on in Figure 6 (left column). After this preparation step, the tomography acquisition was launched according to the experimental parameters listed in table 1.
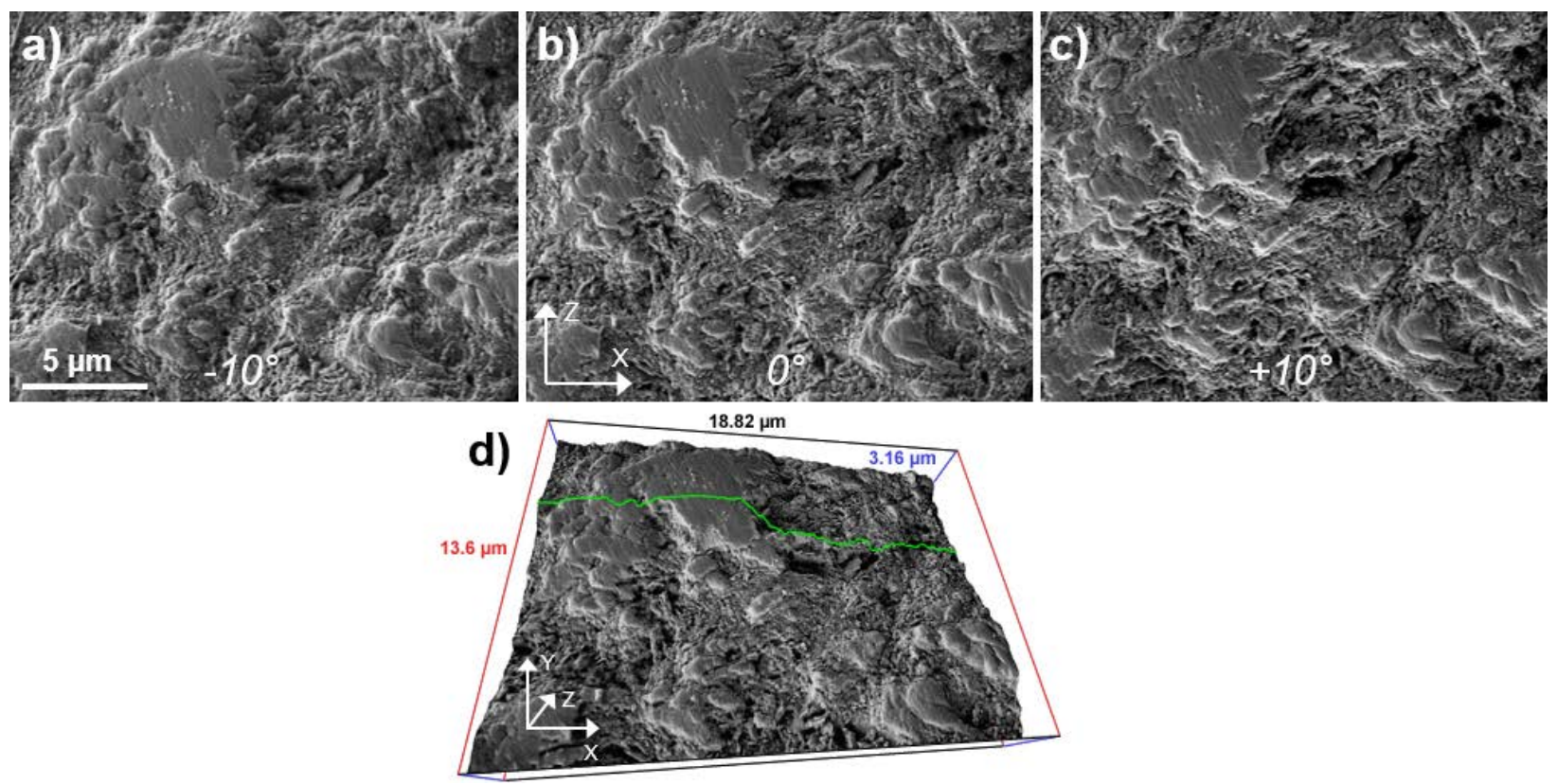

Figure 5. Reconstruction of the sample surface with the MEX software. a-c): SEM images of the surface tilted at $-10,0$ and $+10^{\circ}$ respectively (tilt axis vertical). d): reconstructed surface; the superimposed green line (with an extension of its height by a factor 2 for a better visibility) is one of the MEX profiles used to align the FIB series, see Figure 6. 
Table 1. Experimental conditions for topographic experiment. The INC STYLE parameter defines the targeted slice thickness.

\begin{tabular}{lclc}
\hline \multicolumn{2}{c}{ FIB } & \multicolumn{2}{c}{ SEM } \\
\hline INC STYLE & $10 \mathrm{~nm}$ & SEM pixel size & $10 \mathrm{~nm}$ \\
Milling probe & $700 \mathrm{pA}$ & Aperture size & $60 \mu \mathrm{m}$ \\
Milling depth & $15 \mu \mathrm{m}$ & Detectors & Inlens, EsB \\
FIB EHT & $30 \mathrm{kV}$ & SEM EHT & $1.5 \mathrm{kV}$ \\
\hline
\end{tabular}

Once the whole image stack has been acquired, the alignment procedure will consist in determining the $\mathrm{X}$ and $\mathrm{Y}$ shifts of each image compared to a fixed reference. To do so, the FIB top surface profile of each image of the FIB-SEM stack was extracted by segmenting the high brightness tungsten layer deposited before slicing. Such profiles were then sequentially compared to MEX profiles such as shown in Figure $5 d$. The comparison consists in minimising the Euclidian distance by adjusting the (X, Y, Z) drifts between the each FIB profile and MEX profiles (Z being defined by the rank of the MEX profiles). During this numerical procedure, care was taken to preserve the consistency of length calibration (in pixels or nanometres) in all 3 directions (X, Y, Z). Results are illustrated by Figure 6 through the selection of three slices extracted respectively near the beginning, the middle and the end of the FIB/SEM series. The sensitivity and accuracy of the procedure is further documented by Figure SI-1 in the Supplementary Information.

In addition to the drift corrections in the (X, Y) plans, this surface alignment method also allows to align the sequence in the $\mathrm{Z}$ slicing direction. In a first analysis, no constraint was applied when matching the FIB profile from the $\mathrm{j}^{\text {th }}$ SEM slice with MEX profiles, except that the rank of the best MEX profile BM(i) was forced to be equal or greater to that of the MEX profile matching the (i-1) ${ }^{\text {th }}$ FIB profile. Corresponding results are reported on Figure 7 as 'Absolute match' results (circular symbols). This plot shows that the first 40 FIB profiles are matched by almost the same MEX profiles with an index close to 380. This is due to the relatively flat topography visible on Figure 5d, which does not allow discerning easily close FIB/MEX profiles. The situation gets better for the rest of the series which shows that there is a monotonous variation of BM(i) as a function of $i$. Although monotonous, this variation is however not perfectly linear as it is expected if the slice thickness was constant. It is indeed frequent that fluctuations occur during a FIB/SEM tomography experiment, which induces some variation of this thickness. Moreover, the linear regression of these data exhibits a slope of 0.6, which means that the average interval between MEX profiles matching successive FIB profiles is 0.6, meaning 6 MEX profiles every 10 FIB slices in average. Since the pixel size in SEM images used to obtain the MEX profiles is $10 \mathrm{~nm}$, this indicates that the mean value of INC STYLE is indeed $6 \mathrm{~nm}$ instead of the $10 \mathrm{~nm}$ targeted as reported in Table 2. From a statistical point of view, it is then quite meaningful to re-analyse the data (that is performing again the matching procedure of the whole FIB series) by adding the constraint that the slice thickness is actually constant. Details of this analysis are reported in the SI file, see Figure SI-2, and the result is illustrated by the continuous line 'Match with a constrained slice thickness' 
in Figure 7. Note that this linear variation is obviously close to the linear approximation of the initial matches. The FIB slice thickness is then about $5.75 \mathrm{~nm}$ instead of the $10 \mathrm{~nm}$ value expected according to the setting of the INC STYLE parameter.
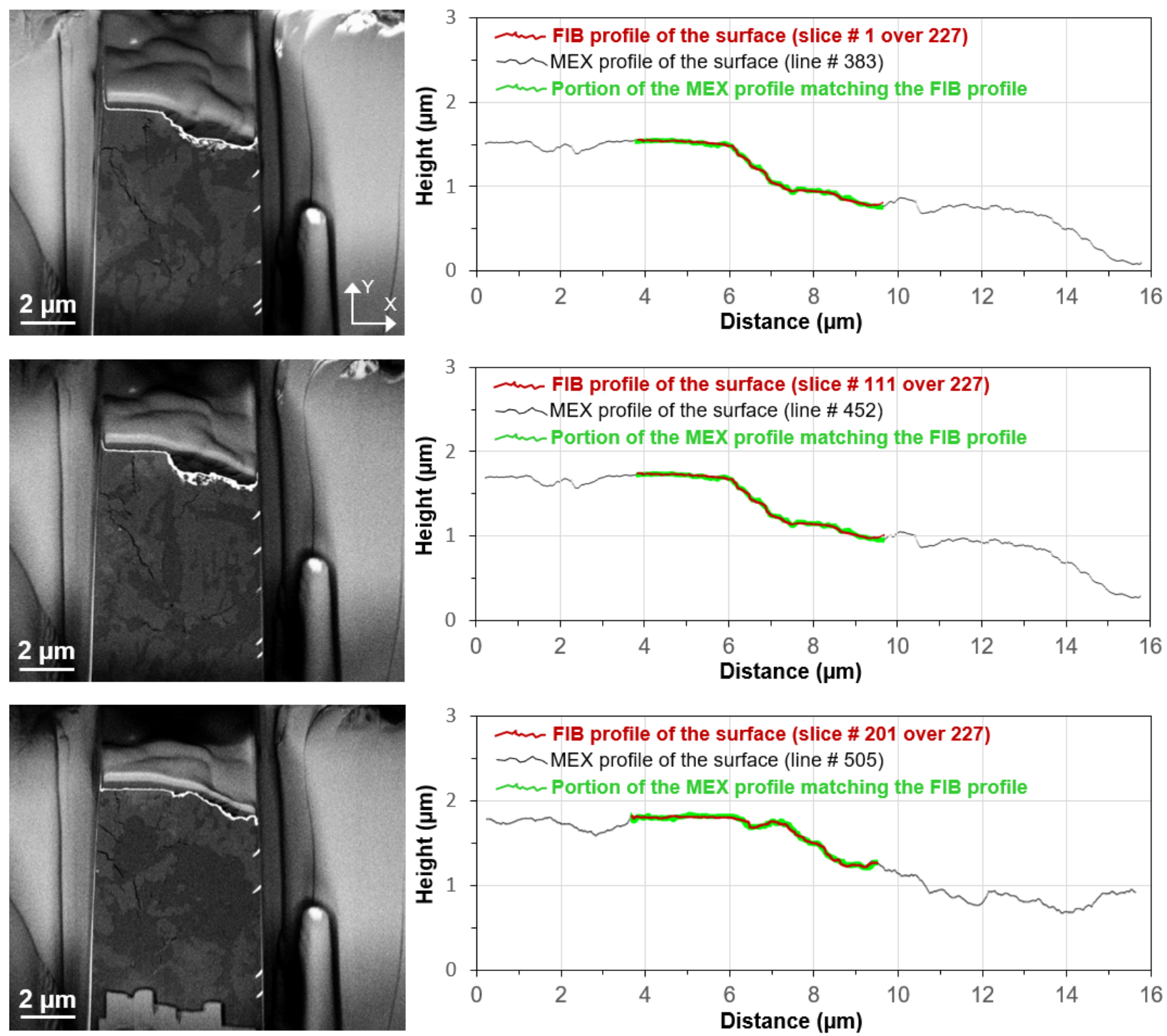

Figure 6. Illustration of the FIB-SEM alignment procedure using the sample surface topography. Left column: selection of three representative (X, Y) SEM micrographs at the beginning, middle and end of the slice-and-view sequence. Right column: corresponding FIB profiles matched by the best MEX profiles according to the minimisation of the Euclidian distance between both curves.

From this complete alignment using the surface topography, we can perform the volume reconstruction and check the lateral side of this volume where line markers were produced prior to the slice-and-view acquisition. Figure 8 shows the fiducial markers milled on the lateral right side; the value of the angle between the two intersecting lines at the bottom of the micrograph in is close to the expected value of $30^{\circ}$ as reported in a). In b), the same side of the sample is shown 
after the reconstruction using the topographic alignment but assuming an isotropic voxel side, meaning a value of INC STYLE as reported in Table 1, i.e. $10 \mathrm{~nm}$. The same angle is measured to about $15^{\circ}$. When correcting INC STYLE to $5.75 \mathrm{~nm}$ according to the 'Match with a constrained slice thickness' performed in Figure 7, the resulting angle get closer to the expected one. This observation is very important, since it means that the slice thickness has to be measured in order to allow accurate and relevant true dimensional measurements on the reconstructed volume.

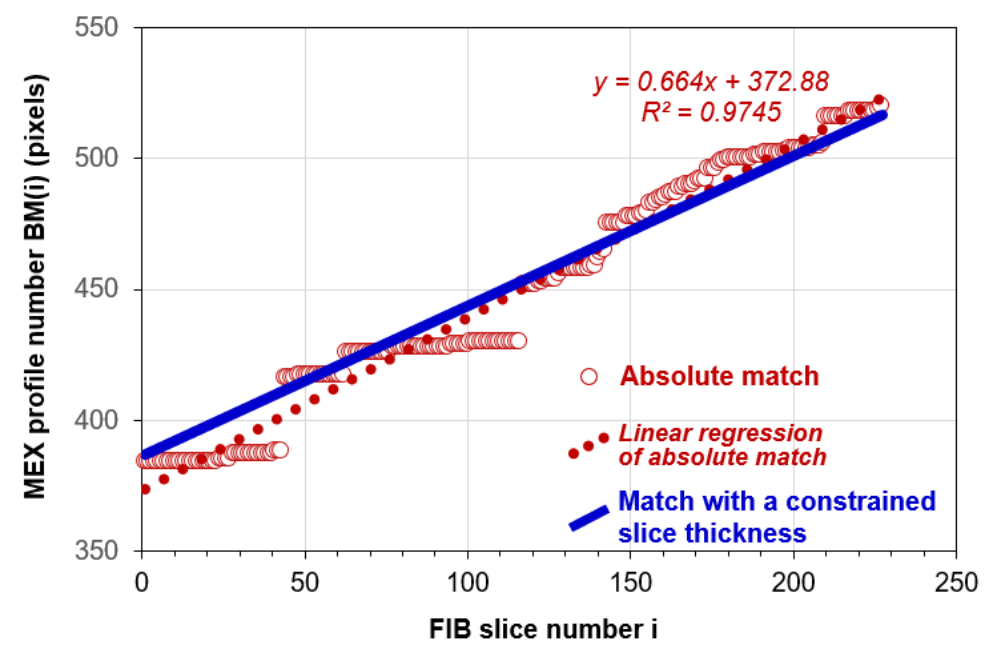

Figure 7. Correspondence between the FIB and MEX profiles: plot of the index of the MEX profile providing the best match of the $\mathrm{i}^{\text {th }}$ FIB profile, $\mathrm{BM}(\mathrm{i})$, as a function of the FIB profile index $\mathrm{i}$ (see text for details).
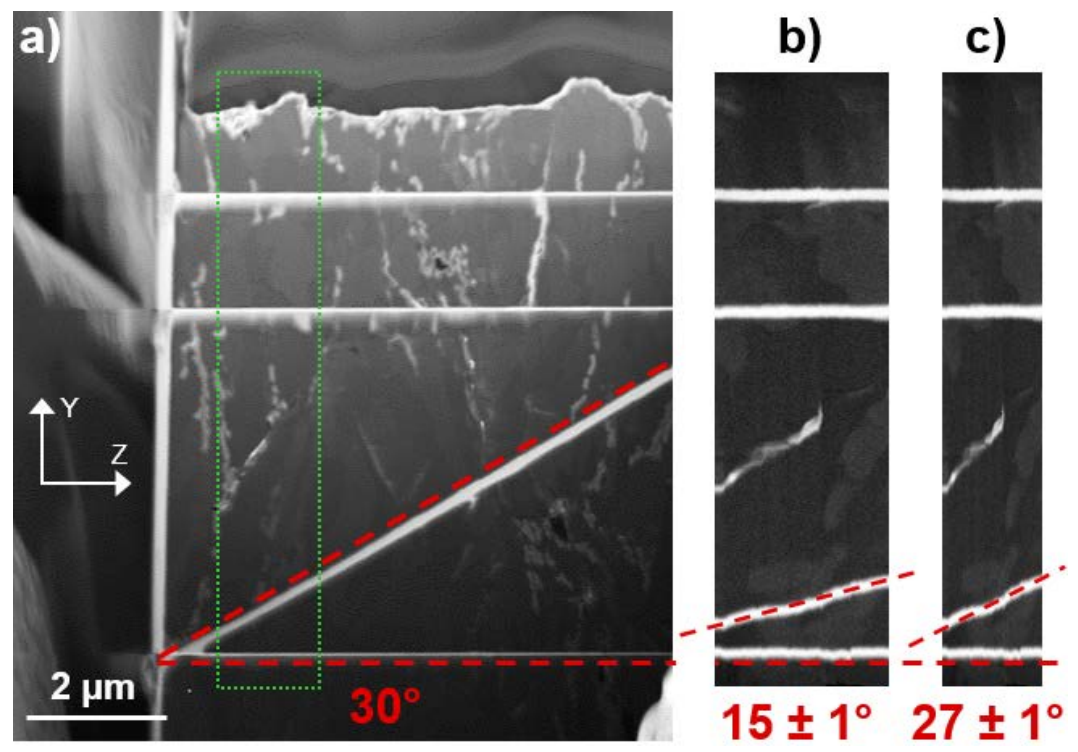

Figure 8. a) View of the fiducial markers milled on the right lateral side of the ROI prior to the slice-andview acquisition; note that the angle between the crossing lines at the bottom is equal to $30^{\circ}$ as indicated. b-c): Same view (detail of the dotted frame in a)) after the topographic reconstruction aligned from the surface topography (averaged projection of 2 orthoslices): b) assuming a pixel size of $10 \mathrm{~nm}$ along $\mathrm{Oz}$ (INC STYLE value), the previous angle is measured at $15 \pm 1^{\circ}$; when correcting INC STYLE to $5.75 \mathrm{~nm}$ (c), the angle is much closer to the expected value. 
At this stage, it must however be stated that both the difference in the angle between intersecting markers and the fluctuations in their linearity easily observed on Fig. 8c) may be considered as, still, an imperfect alignment. This is indeed not the case: it is easily seen that the top horizontal lines are almost perfectly restored and linear, meaning that the alignment is quite good in the upper part of the images. This indicates that the apparent misalignment of the lower part is due to fluctuations in the scan process during acquisition, leading to some random stretching effects along the vertical direction of all micrographs. This phenomenon has been encountered many times during numerous 3D acquisitions, whatever the type of samples (conducting or non-conducting, sensitive to the electron beam or not,...) and was also previous evoked in the literature [38]. At this stage, we cannot ascertain whether this stretching effect is due to some external perturbation, such as a stray field, or to internal problems, such as slight magnification changes caused by some lens hysteresis for example. It may be thought that a more sophisticated control of the electron beam scanning, as permitted for example by the external Fibics Inc. device could improve the situation.

To conclude on this part, we can say that the topographic alignment performed here gives very good results; it has now to be compared quantitatively compared to alternative and more classical alignments methods as described in the section 3.2.

\subsubsection{Comparing the topographic alignment with other alignment methods}

We have already demonstrated that direct cross-correlation based alignments on the microstructure are not accurate (see section 3.2). Nevertheless, it is still worth comparing these methods with the topographic alignment described above. Additional alignments were thus performed by cross-correlation using the plugin 'MultiStackReg' in the Fiji software for three different areas: 1) the two parallel upper lines shown in Figure 8 but when seen edge-on from the viewing $\mathrm{Z}$ direction as in Figure 6, 2) the interface between the deposited $\mathrm{W}$ and $\mathrm{C}$ layers and the sample top surface and 3) the centre of the ROI.

Figure 9 provides the comparison between reconstructed volumes using all approaches (see also Figure SI-3 of the S.I. file). An error factor is reported on each display: it corresponds to the mean Euclidian distance $\mathrm{E}$ between both surfaces per pixel (i.e., the sum of distances between both reconstructed surfaces for all pixels divided by the number of pixels of the MEX top surface).

The main conclusion is that the topographic alignment (Figure 9a) exhibits a good quality as does the 'two markers' alignment (Figure 9b). Nevertheless, the faint contrast of the top markers in Fig. 9b) indicates a slight misalignment in the $\mathrm{X}$ direction.

Whereas it is obvious that the two last approaches (cross-correlation directly on the sample microstructure, even including the top surface intersection - Figure 9c) -) do not provide correct alignments, it is shown that using the lateral top marker lines leads to a reasonable alignment, although quantitatively slightly worse than the one based with the surface topography. All these reconstructions suffer however from the scan distortions illustrated by the wavy shape of the bottom markers and remain improvable. 

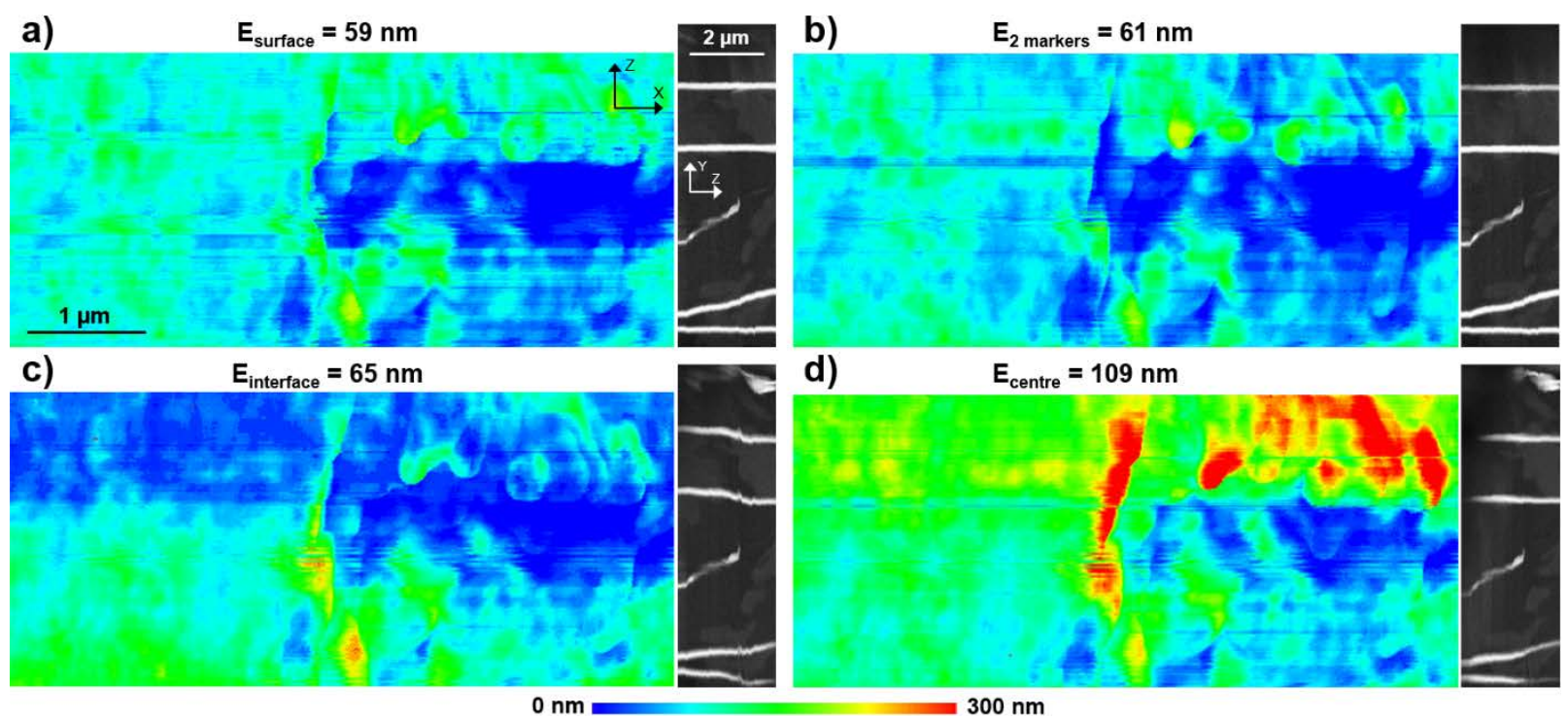

Figure 9. Top view of the differences between the reconstructed surface with various alignment procedures and the MEX surface: a) the surface topography, b) the two parallel markers on the top of the lateral righthand side, c) the interface between the top surface and the deposited layers, d) a central (X, Y) area of the ROI (b) to d): cross-correlation method). In each case, the reconstructed lateral side is shown as in Figure $8 \mathrm{~b}$ (for a better visibility, the pixel size of $10 \mathrm{~nm}$ in the $\mathrm{Z}$ direction has not been corrected). The difference in height between the two surfaces at each pixel is shown in a normalized false colours look-up-table representing the difference in height $(\mathrm{nm})$ between each reconstructed surface and the MEX reference. The mean Euclidian distance by pixel is indicated in each case, it has been calculated on a cropped surface size identical for all methods. Note that horizontal shears occur in each case, which indicate imperfect alignments of numerous frames of the slice-and-view series.

\subsection{Drilled tubular hole}

It was already stated that markers that are commonly machined on the surfaces of the samples may not be good references for the alignment, since depending on the surface topography, and/or partially erased or modified during the FIB process due to degradation and/or re-deposition under the effect of the ion beam. Therefore, we need a marker which can serve as an absolute geometrical reference insensitive to such modifications.

In Figure 2, we have discussed the problem of aligning an inclined 'tube', which would be transformed as a horizontal one if no information is provided on its inclination. This constitutes the basis of a new marker type: we can FIB-drill a hole across the ROI, and knowing its angle of inclination and the distance between successive slices (FIB slicing step), a reasonable alignment can be attempted. The idea of drilling holes to serve as alignment markers was already proposed [45], but with a different geometry. From this idea, a hole across all the volume to be sliced-andviewed has been performed as illustrated in Figure 10. We used the same sample as for the previous experiment described in section 4.1. Typically, the sample is tilted at $54^{\circ}$ in the microscope to reach the FIB position (i.e. the ion beam arrives perpendicular to the sample surface). In order to drill a tube before the acquisition, the stage is tilted at $0^{\circ}$, leading to a hole axis inclined by $36^{\circ}$ with respect to the top surface of the sample, as depicted in Figure 10a. Then, the ion beam is set 
to the spot mode at a given position point $\mathrm{E}$ on the sample entrance surface as shown in Figure 10b, closed to the working area for a sufficiently long time in order to drill a hole through a reasonable thickness, namely the thickness that will be removed during the forthcoming 3D acquisition. In our attempts, we found it reasonable to use about 8 minutes according to the experimental parameters listed in table 1, i.e. a FIB current of 700 pA. An important parameter is the defocusing of the beam, which is also required to perform an efficient drilling: if the beam is focussed on the top surface, a large crater is created but the hole drilling is not directional as required for the purpose of the experiment. Then a tomographic series of images was acquired, and the alignment using the surface topography was also performed for comparison purposes.

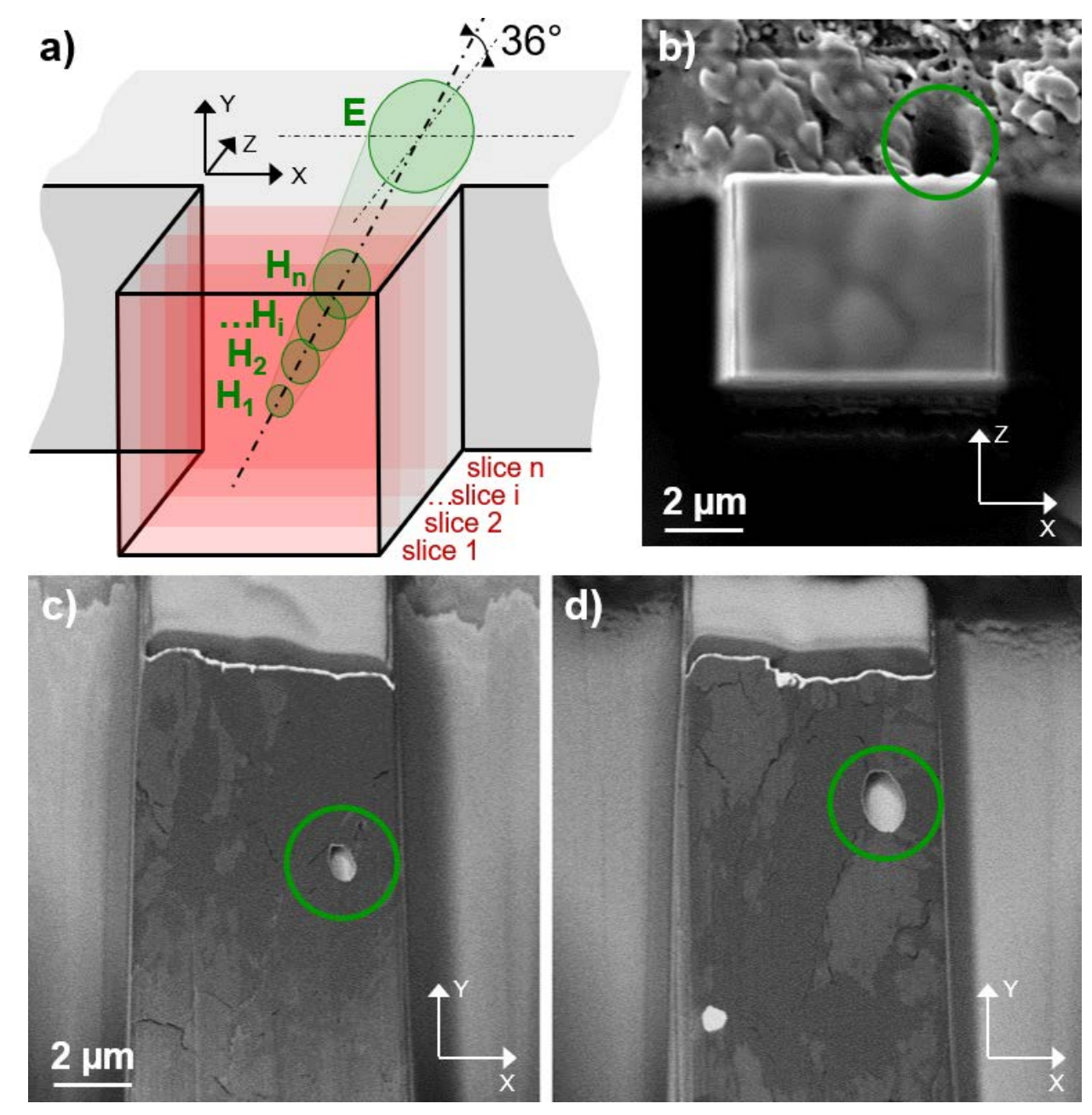

Figure 10. Hole drilling before the FIB/SEM tomographic acquisition. a): Schematic view of the geometry. b): View of the entrance of the hole E from the top surface. c)-d): SEM images taken at the start of the sliceand-view sequence showing the holes intersecting the first and last slices (labelled respectively $\mathrm{H}_{1}$ and $\mathrm{H}_{\mathrm{n}}$ in a).

The post-mortem 'hole using' alignment was performed according to the following procedure. The image stack was first aligned through a cross-correlation method applied to a (X, Y) ROI 
limited to the intersection of the drilled hole in all slices (hole sections labelled $\mathrm{H}_{\mathrm{i}}$ in Figure 10a). This leads to a horizontal linear hole axis as expected from the illustration in Figure 2. The image stack was then projected on the $(\mathrm{Y}, \mathrm{Z})$ plane and successive images were subsequently shifted along $\mathrm{Y}$ to reproduce the theoretical inclination angle of $36^{\circ}$ as mentioned above. The resulting volume is depicted in Figure 11a-c); for a better visibility, only the hole trace and the top surface underlined by the tungsten layer used for the topographic alignment are shown in a).
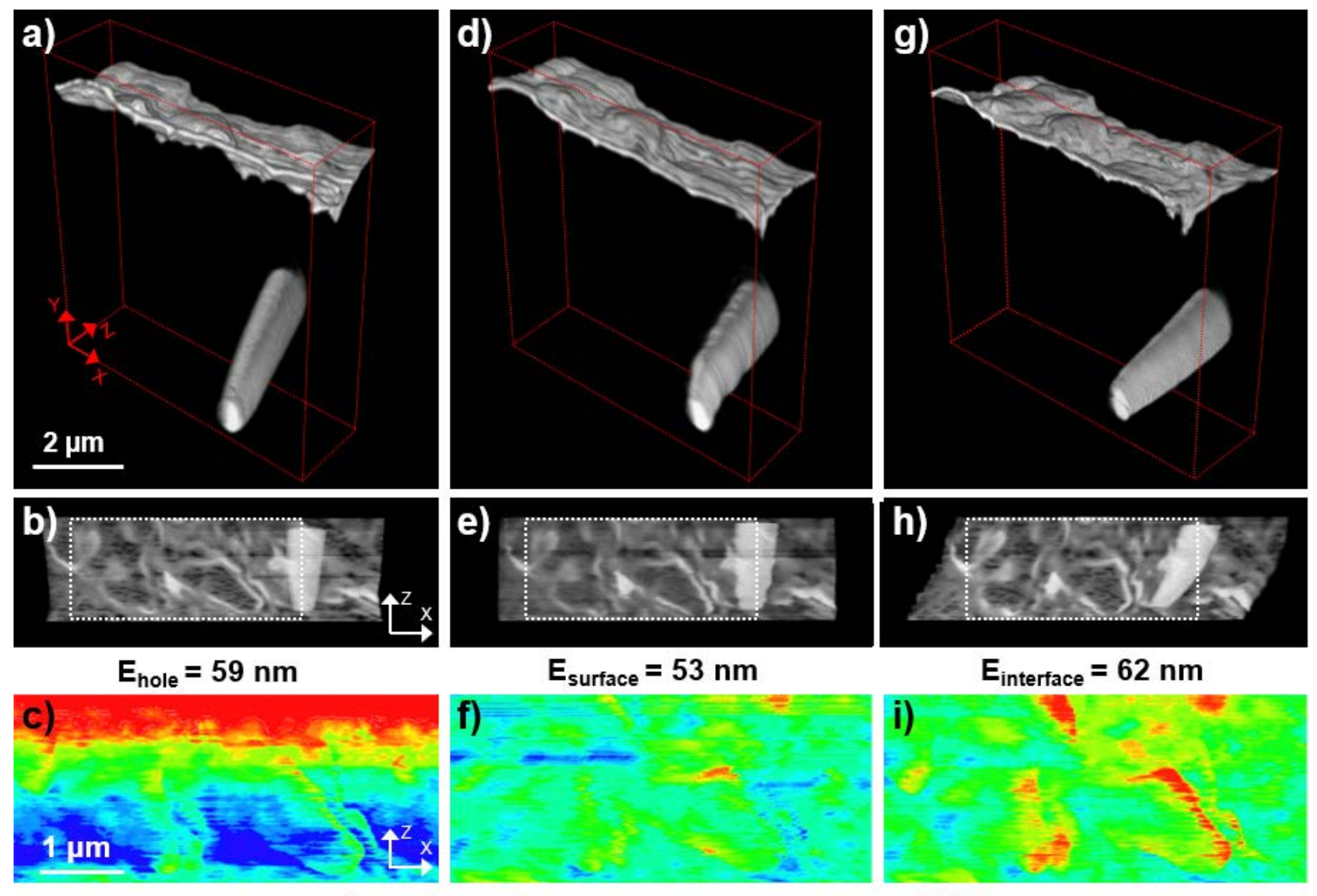

$0 \mathrm{~nm}$

$200 \mathrm{~nm}$

Figure 11. 3D perspective of the deconstructed drilled hole and sample surface after aligning the FIB-SEM stack of images. a): Alignment performed by using the hole sections; b): top view of the upper surface after reconstruction (partial transparency 75\%); c) display, in the reference area defined by the dotted white frame in b), of the difference in height between the reconstructed surface and the MEX reference shown in a normalized false colours look-up-table as in Figure 9. d-f): Similar results obtained from a topographic alignment as described in section 4.1. g-i): Similar results after a cross-correlation alignment over a selected ROI including the interface and the top surface. The mean Euclidian distance by pixel is indicated in c), $\mathrm{f}$ ) and i); it has been calculated over the areas of colour maps c), f) and i).

Other illustrations in Figure 11 are for results obtained after aligning the image series using the topography method (Figure 11d-f) and a classical cross-correlation of a (X, Y) ROI (Figure 11gi) selected such as it includes the top surface of the reconstructed area. 3D views in Fig. 11a,d,g) are drawn in a very similar orientation. 
The best result remains that using the surface topography alignment. The Euclidian distance between the reconstructed top surface and that calculated from the MEX analysis is minimal, which confirms that both surfaces are closely inter-penetrating, then close one to each other as suggested by the composite images reported in Figure SI-4.

Indeed, the manual alignment using the drilled hole gives a reasonable result; this will be further discussed in section 5 . Figure 12 shows $(\mathrm{Y}, \mathrm{Z})$ orthoslices from the reconstructed volumes around the central section of the tubular hole; this illustrates qualitatively the ranking of the three alignment methods in terms of quality. Note that the slice thickness deduced from the topography alignment was again found to be close to $0.6 \mathrm{~nm}$. This parameter is indeed essential to allow a correct inclination of the reconstructed hole as shown in Figure 12a.

In other words, the correct alignment based on a tubular hole relies on the accuracy of value of the slice thickness normally defined the INC STYLE parameter in Table 1, which in the present case requires an independent method to evaluate this parameter.

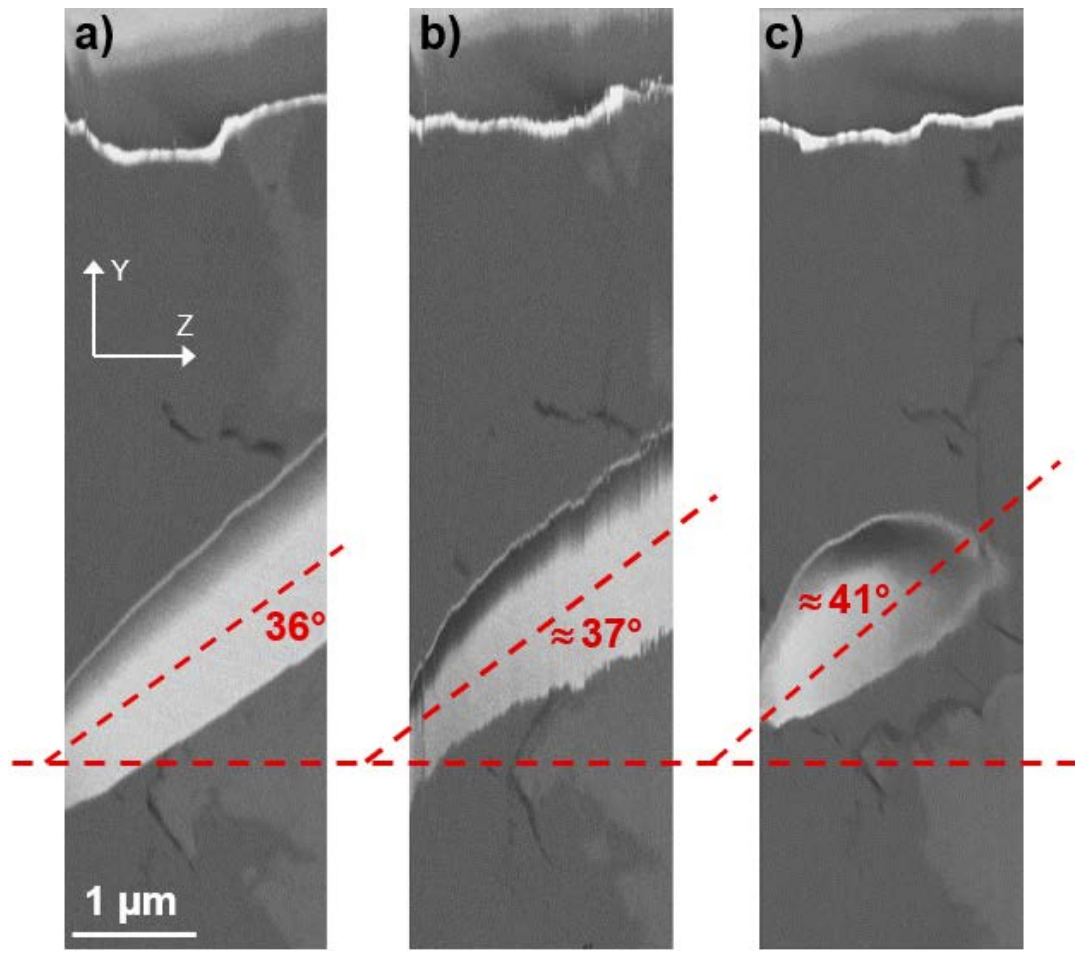

Figure 12. Lateral (Z, Y) views of the median section of reconstructed volumes showing the hole corresponding to Figures 10a-d 11. a): Results from the alignment performed by forcing the average inclination of the hole to the expected value, i.e. $36^{\circ}$. b): Same as a) when the alignment was performed using the top surface topography). c): Same as a) and b) when the alignment was performed using the crosscorrelation method). In all cases angle values are the mean inclination of the hole axis (see text for details).

All this analysis further confirms that the alignment based on a cross-correlation of a (X, Y) ROI selected from the image series does not give excellent results. This alignment gives a rather inaccurate estimation of the inclination of the drilled hole, which remains however comparable to the expected value (Figure 12c). Moreover, it is seen that the hole envelope is not complete since 
it disappears at the right-hand side of the projection, that is towards the end of the images stack. This effect is a consequence of significant shifts along the $\mathrm{X}$ direction evidenced in Figure 11h; the presence of such shifts also produces the large red areas in Figure SI-4c), proving that the FIB reconstructed to surface appears higher that the MEX surface.

\section{Discussion and conclusions}

\subsection{Discussion}

The present work aimed at discussing the methods that can be used in order to produce a reasonable alignment of the image stack obtained after such a 3D FIB-SEM experiment. This task is generally considered as an easy one, since, in principle, only $\mathrm{X}$ and $\mathrm{Y}$ (small) shifts of the working area are expected. One origin of these shifts can be some instability during the experiment itself, and it is tempting to try to correct them in situ as was proposed [30]. Then, one may expect that a cross-correlation method can simply be used in order to align the image stack.

We have shown here that it is not true. Cross-correlation method is a powerful method to align images from a numerical point of view, but since they do not rely on an accurate 'a priori' knowledge of the true 3D microstructure, they cannot give universally good results. Even when using fiducial markers pre-machined on the lateral surfaces of the working area, some undesirable misalignments can subsist, which are indeed undetectable if no universal reference is available (the majority of the cases). Therefore, a post-mortem alignment is needed even if some alignment is performed in situ with the intention to roughly keep the ROI in the field-of-view.

We have then demonstrated that using the topography of the sample surface itself, possibly reconstructed from a stereoscopic analysis (as, for example, permitted by commercial softwares such as MEX which was used in the present work), can be a good reference providing the mean of a correct alignment. To do so, we have developed a method consisting in aligning line profiles extracted from the SEM- FIB images stack with corresponding ones obtained from the 'MEX' surface reconstruction. This approach has also allowed us to provide a way to estimate more accurately the thickness of the slices of matter removed during the $3 \mathrm{D}$ acquisition. Indeed, the method provides a good means for relatively correct alignments along the 3 directions $\mathrm{X}, \mathrm{Y}$ and Z.

The method is efficient but suffers some limitations.

The main difficult relies in the topography of the top surface itself, which must vary sufficiently to allow a good reconstruction, and consequently a good alignment. Then line profiles must be extracted from the intersection of this surface with each image of the FIB-SEM stack. This can be performed by depositing a heavy element, tungsten or platinum on the surface and prior to a carbon protection layer, the SEM contrast of which is adequate for profile extraction by standard image segmentation as reported in Figure 6. Some accidents at the surface may limit the accuracy with which these profiles can be re-associated to surface line profiles reconstructed by a stereophotogrammetry approach. For example, small open pores at the surface may lead to 
thickening of the deposited layer, giving rise to extracted profiles with some uncertainty in their height owing to their thickness; such difficulties were encountered near the middle of the top surface as seen in the micrographs shown in Figure 6.

Another drawback of the method is that it is time consuming. Whereas the stereophotogrammetry analysis can be relatively fast (two or three SEM images to record before fibbing and a rapid reconstruction with a dedicated software such as MEX), the extraction of FIB profiles and their association to MEX line profiles requires a long post-treatment which should be optimized from a software point of view in order to allow its routine application.

We have also tested an alternative method consisting to drill a hole across the ROI in order to constitute a good reference for a subsequent alignment of the image stack. It can certainly be further improved especially to try to obtain a constant diameter of the hole, which appeared here to vary as a function of depth. Although the quality of this alignment has been shown to be reasonable, it can be questioned, since noticeable misalignments may be induced by several factors: (i) if the hole sections in each slice is not isotropic, the vertical alignment of the hole axis as described in section 4.2 may be flawed; in addition, the hole size varies along the successive slices, which may hamper an accurate alignment. (ii) When adjusting the deduced inclination angle of $36^{\circ}$ in the $(\mathrm{Y}, \mathrm{Z})$ projection, errors may occur if the original axis of the hole was not strictly linear, due for example to significant drift during drilling. This is eventually what may happen at the beginning of the FIB sequence: the topography alignment produces an inclination of the hole axis towards the bottom at the beginning of the sequence, i.e. at the end of the hole drilling. This deviation is obviously corrected by the manual alignment of the hole axis, but this correction remains subjective and possibly flawed. Moreover, the method is not very convenient since it suffers from other drawbacks: firstly, the hole is essentially in the middle of the area of interest, which implies a lack of information about the reconstructed microstructure, and possibly some damage in its vicinity. Secondly, drilling the hole, although quite rapidly machined under the present conditions, requires some skill in order to realize it in the adequate incidence and position. Thirdly, the accuracy of the alignment of the hole axis along the correct inclination (i.e. $36^{\circ}$ in the present case) is strongly dependent on the accuracy of the slice thickness measurement, as well as its reproducibility. We have seen here that this value varied quite a long during the slicing sequence and that the targeted value (the INC STYLE parameter in Table 1) cannot necessarily be trusted: the topography alignment confirmed that this parameter was $40 \%$ lower than the expected value (about $6 \mathrm{~nm}$ compared to an expectation of 10).

\subsection{Summary}

The present wok was initiated to examine the possibilities and limits of alignments of image series acquired during FIB-SEM tomography, and especially post-mortem final alignments. Main results are:

(i) Extracting a small volume from the surface of a bulk sample has made it possible to mill different line markers on the lateral side easily accessible to the ion beam, thus allowing to 
test various alignments methods based on cross-correlation (section 3). This study demonstrates, as many previous works did [35,37,38], that such methods are not accurate

(ii) We have developed a methodology to align the FIB-SEM sequence using the topography of the top surface of the sample itself. It is based on a prior determination of the surface topography by stereophotogrammetry, line profiles of which allow a complete $(\mathrm{X}, \mathrm{Y}, \mathrm{Z})$ alignment of the FIB slices. Demonstration of the efficiency of the method was performed using a mineral from the earth crust, i.e. serpentine.

(iii) The topography alignment method showed its global superiority to other alignments methods, including X-correlation based ones and another tested approach relying on the alignment of a hole intentionally drilled across the volume of interest.

(iv) Although powerful, the topography method certainly needs further improvements for being applied routinely, especially, speeding up the analysis and improving its accuracy.

\section{Acknowledgments}

The CLYM (www.clym.fr) is gratefully acknowledged for the access to the FIB instrument. This work was partially funded by Carl Zeiss S.A.S. in a PhD study. We thank N. Hilairet and B. Reynard from the Laboratoire de Géologie de Lyon : Terre, Planètes, Environnement (LGL-TPE, UMR CNRS 5276 ENS Lyon) for providing the mineral used in this study. S. Descartes from the Contact \& Structure Mechanics laboratory (LaMCos, UMR CNRS 5259, INSA Lyon) is kindly acknowledged for the access to MEX software. This paper is dedicated to our dear colleague and co-author BVDM who was one of the responsible persons in charge of the FIB instrument at CLYM and who passed away from cancer disease on January 2, 2015 at the age of 42 ans.

\section{References}

[1] E. Maire, P.J. Withers, Quantitative X-ray tomography, Int. Mater. Rev. 59 (2013) 1-43. https://doi.org/10.1179/1743280413y.0000000023.

[2] F. Pfeiffer, X-ray ptychography, Nat. Photonics. 12 (2018) 9-17. https://doi.org/10.1038/s41566-017-0072-5.

[3] M. Du, Y.S.G. Nashed, S. Kandel, D. Gürsoy, C. Jacobsen, Three dimensions, two microscopes, one code: Automatic differentiation for x-ray nanotomography beyond the depth of focus limit, Sci. Adv. 6 (2020). https://doi.org/10.1126/sciadv.aay3700.

[4] P.A. Midgley, M. Weyland, 3D electron microscopy in the physical sciences: The development of Z-contrast and EFTEM tomography, Ultramicroscopy. 96 (2003) 413- 
431. https://doi.org/10.1016/S0304-3991(03)00105-0.

[5] P. Ercius, O. Alaidi, M.J. Rames, G. Ren, Electron Tomography: A Three-Dimensional Analytic Tool for Hard and Soft Materials Research, Adv. Mater. 27 (2015) 5638-5663. https://doi.org/10.1002/adma.201501015.

[6] B. Goris, S. Bals, W. Van Den Broek, E. Carbó-Argibay, S. Gómez-Graña, L.M. LizMarzán, G. Van Tendeloo, Atomic-scale determination of surface facets in gold nanorods, Nat. Mater. 11 (2012) 930-935. https://doi.org/10.1038/nmat3462.

[7] J. Zhou, Y. Yang, P. Ercius, J. Miao, Atomic electron tomography in three and four dimensions, MRS Bull. 45 (2020) 290-297. https://doi.org/10.1557/mrs.2020.88.

[8] X. Tian, D.S. Kim, S. Yang, C.J. Ciccarino, Y. Gong, Y. Yang, Y. Yang, B. Duschatko, Y. Yuan, P.M. Ajayan, J.C. Idrobo, P. Narang, J. Miao, Correlating the three-dimensional atomic defects and electronic properties of two-dimensional transition metal dichalcogenides, Nat. Mater. (2020). https://doi.org/10.1038/s41563-020-0636-5.

[9] A. Devaraj, D.E. Perea, J. Liu, L.M. Gordon, T.J. Prosa, P. Parikh, D.R. Diercks, S. Meher, R.P. Kolli, Y.S. Meng, S. Thevuthasan, Three-dimensional nanoscale characterisation of materials by atom probe tomography, Int. Mater. Rev. 63 (2018) 68101. https://doi.org/10.1080/09506608.2016.1270728.

[10] S.M. Reddy, D.W. Saxey, W.D.A. Rickard, D. Fougerouse, S.D. Montalvo, R. Verberne, A. van Riessen, Atom Probe Tomography: Development and Application to the Geosciences, Geostand. Geoanalytical Res. 44 (2020) 5-50. https://doi.org/10.1111/ggr.12313.

[11] L. Holzer, F. Indutnyi, P. Gasser, B. Munch, M. Wegmann, Three-dimensional analysis of porous BaTiO3 ceramics using FIB nanotomography, J. Microsc. 216 (2004) 84-95. https://doi.org/10.1111/j.0022-2720.2004.01397.x.

[12] M. Cantoni, L. Holzer, Advances in 3D focused ion beam tomography, MRS Bull. 39 (2014) 354-360. https://doi.org/10.1557/mrs.2014.54.

[13] J.A. Taillon, C. Pellegrinelli, Y.L. Huang, E.D. Wachsman, L.G. Salamanca-Riba, Improving microstructural quantification in FIB/SEM nanotomography, Ultramicroscopy. 184 (2018) 24-38. https://doi.org/10.1016/j.ultramic.2017.07.017.

[14] R. Wirth, Focused Ion Beam (FIB) combined with SEM and TEM: Advanced analytical tools for studies of chemical composition, microstructure and crystal structure in geomaterials on a nanometre scale, Chem. Geol. 261 (2009) 217-229. https://doi.org/10.1016/j.chemgeo.2008.05.019.

[15] Y. Liu, H. King, M. van Huis, M. Drury, O. Plümper, Nano-Tomography of Porous Geological Materials Using Focused Ion Beam-Scanning Electron Microscopy, Minerals. 6 (2016) 104. https://doi.org/10.3390/min6040104.

[16] Y. Hua, P. Laserstein, M. Helmstaedter, Large-volume en-bloc staining for electron microscopy-based connectomics, Nat. Commun. 6 (2015) 1-7. 
https://doi.org/10.1038/ncomms8923.

[17] C. Kizilyaprak, Y.D. Stierhof, B.M. Humbel, Volume microscopy in biology: FIB-SEM tomography, Tissue Cell. 57 (2019) 123-128. https://doi.org/10.1016/j.tice.2018.09.006.

[18] T.L. Burnett, R. Kelley, B. Winiarski, L. Contreras, M. Daly, A. Gholinia, M.G. Burke, P.J. Withers, Large volume serial section tomography by Xe Plasma FIB dual beam microscopy, Ultramicroscopy. 161 (2016) 119-129. https://doi.org/10.1016/j.ultramic.2015.11.001.

[19] A. Gholinia, M.E. Curd, E. Bousser, K. Taylor, T. Hosman, S. Coyle, M.H. Shearer, J. Hunt, P.J. Withers, Coupled Broad Ion Beam-Scanning Electron Microscopy (BIB-SEM) for polishing and three dimensional (3D) serial section tomography (SST), Ultramicroscopy. 214 (2020) 112989. https://doi.org/10.1016/j.ultramic.2020.112989.

[20] M.P. Echlin, M. Straw, S. Randolph, J. Filevich, T.M. Pollock, The TriBeam system: Femtosecond laser ablation in situ SEM, Mater. Charact. 100 (2015) 1-12. https://doi.org/10.1016/j.matchar.2014.10.023.

[21] M. Groeber, B. Haley, M. Uchic, S. Ghosh, Microstructural Characterization Using 3-D Orientation Data Collected by an Automated FIB-EBSD System, in: AIP Conf. Proc., AIP, 2004: pp. 1712-1718. https://doi.org/10.1063/1.1766778.

[22] J. Konrad, S. Zaefferer, D. Raabe, Investigation of orientation gradients around a hard Laves particle in a warm-rolled Fe3Al-based alloy using a 3D EBSD-FIB technique, Acta Mater. 54 (2006) 1369-1380. https://doi.org/10.1016/j.actamat.2005.11.015.

[23] A. Fernández, A. Jérusalem, I. Gutiérrez-Urrutia, M.T. Pérez-Prado, Three-dimensional investigation of grain boundary-twin interactions in a Mg AZ31 alloy by electron backscatter diffraction and continuum modeling, Acta Mater. 61 (2013) 7679-7692. https://doi.org/10.1016/j.actamat.2013.09.005.

[24] P.G. Kotula, M.R. Keenan, J.R. Michael, M icroscopy M icroanalysis Tomographic Spectral Imaging with Multivariate Statistical Analysis : Comprehensive 3D Microanalysis, (2018) 36-48.

[25] M. Schaffer, J. Wagner, B. Schaffer, M. Schmied, H. Mulders, Automated threedimensional X-ray analysis using a dual-beam FIB, Ultramicroscopy. 107 (2007) 587597. https://doi.org/10.1016/j.ultramic.2006.11.007.

[26] P. Burdet, J. Vannod, A. Hessler-Wyser, M. Rappaz, M. Cantoni, Three-dimensional chemical analysis of laser-welded NiTi-stainless steel wires using a dual-beam FIB, Acta Mater. 61 (2013) 3090-3098. https://doi.org/10.1016/j.actamat.2013.01.069.

[27] D.J. Child, G.D. West, R.C. Thomson, The use of combined three-dimensional electron backscatter diffraction and energy dispersive $\mathrm{X}$-ray analysis to assess the characteristics of the gamma/gamma-prime microstructure in alloy $720 \mathrm{Li}^{\mathrm{TM}}$, Ultramicroscopy. 114 (2012) 1-10. https://doi.org/10.1016/j.ultramic.2011.11.003.

[28] T. Dahmen, M. Engstler, C. Pauly, P. Trampert, N. De Jonge, F. Mücklich, P. Slusallek, 
Feature Adaptive Sampling for Scanning Electron Microscopy, Sci. Rep. 6 (2016) 1-11. https://doi.org/10.1038/srep25350.

[29] C.S. Xu, K.J. Hayworth, Z. Lu, P. Grob, A.M. Hassan, J.G. García-Cerdán, K.K. Niyogi, E. Nogales, R.J. Weinberg, H.F. Hess, Enhanced FIB-SEM systems for large-volume 3D imaging, Elife. 6 (2017) 1-36. https://doi.org/10.7554/eLife.25916.

[30] H. Yuan, B. Van De Moortèle, T. Epicier, J.C. Menard, Live correction of drifts during 3D-FIB experiments, EMC 2012 Proc. 15th Eur. Microsc. Congr. (2012) 135-136. http://www.emc2012.org.uk/documents/Abstracts/Abstracts/EMC2012_0791.pdf.

[31] A. Schertel, N. Snaidero, H.M. Han, T. Ruhwedel, M. Laue, M. Grabenbauer, W. Möbius, Cryo FIB-SEM: Volume imaging of cellular ultrastructure in native frozen specimens, J. Struct. Biol. 184 (2013) 355-360. https://doi.org/10.1016/j.jsb.2013.09.024.

[32] J. Hennies, J.M.S. Lleti, N.L. Schieber, R.M. Templin, A.M. Steyer, Y. Schwab, AMST: Alignment to Median Smoothed Template for Focused Ion Beam Scanning Electron Microscopy Image Stacks, Sci. Rep. 10 (2020) 1-10. https://doi.org/10.1038/s41598-02058736-7.

[33] S. Zaefferer, S.I. Wright, D. Raabe, Three-Dimensional Orientation Microscopy in a Focused Ion Beam-Scanning Electron Microscope: A New Dimension of Microstructure Characterization, Metall. Mater. Trans. A. 39 (2008) 374-389. https://doi.org/10.1007/s11661-007-9418-9.

[34] M. Ritter, P.A. Midgley, A practical approach to test the scope of FIB-SEM 3D reconstruction, J. Phys. Conf. Ser. 241 (2010). https://doi.org/10.1088/17426596/241/1/012081.

[35] H.J.T. Stephensen, S. Darkner, J. Sporring, Restoring drifted electron microscope volumes using synaptic vesicles at sub-pixel accuracy, Commun. Biol. 3 (2020) 81. https://doi.org/10.1038/s42003-020-0809-4.

[36] H. Iwai, N. Shikazono, T. Matsui, H. Teshima, M. Kishimoto, R. Kishida, D. Hayashi, K. Matsuzaki, D. Kanno, M. Saito, H. Muroyama, K. Eguchi, N. Kasagi, H. Yoshida, Quantification of SOFC anode microstructure based on dual beam FIB-SEM technique, J. Power Sources. 195 (2010) 955-961. https://doi.org/10.1016/j.jpowsour.2009.09.005.

[37] H.G. Jones, K.P. Mingard, D.C. Cox, Investigation of slice thickness and shape milled by a focused ion beam for three-dimensional reconstruction of microstructures, Ultramicroscopy. 139 (2014) 20-28. https://doi.org/10.1016/j.ultramic.2014.01.003.

[38] K.R. Mangipudi, V. Radisch, L. Holzer, C.A. Volkert, A FIB-nanotomography method for accurate 3D reconstruction of open nanoporous structures, Ultramicroscopy. 163 (2016) 38-47. https://doi.org/10.1016/j.ultramic.2016.01.004.

[39] J.R. Kremer, D.N. Mastronarde, J.R. McIntosh, Computer visualization of threedimensional image data using IMOD, J. Struct. Biol. 116 (1996) 71-76.

https://doi.org/10.1006/jsbi.1996.0013. 
[40] P. Thevenaz, U.E. Ruttimann, M. Unser, A pyramid approach to subpixel registration based on intensity, IEEE Trans. Image Process. 7 (1998) 27-41.

https://doi.org/10.1109/83.650848.

[41] C. Holzapfel, W. Schäf, M. Marx, H. Vehoff, F. Mücklich, Interaction of cracks with precipitates and grain boundaries: Understanding crack growth mechanisms through focused ion beam tomography, Scr. Mater. 56 (2007) 697-700.

https://doi.org/10.1016/j.scriptamat.2006.12.025.

[42] F. Lasagni, A. Lasagni, M. Engstler, H.P. Degischer, F. Mücklich, Nano-characterization of cast structures by FIB-tomography, Adv. Eng. Mater. 10 (2008) 62-66.

https://doi.org/10.1002/adem.200700249.

[43] B.J. Inkson, T. Steer, G. Möbus, T. Wagner, Subsurface nanoindentation deformation of Cu-Al multilayers mapped in 3D by focused ion beam microscopy, J. Microsc. 201 (2001) 256-269. https://doi.org/10.1046/j.1365-2818.2001.00767.x.

[44] S. Cao, W. Tirry, W. Van Den Broek, D. Schryvers, Optimization of a FIB/SEM sliceand-view study of the 3D distribution of Ni4Ti3precipitates in Ni-Ti, J. Microsc. 233 (2009) 61-68. https://doi.org/10.1111/j.1365-2818.2008.03095.x.

[45] M.D. Uchic, M. De Graef, R. Wheeler, D.M. Dimiduk, Microstructural tomography of a Ni70Cr20Al10 superalloy using focused ion beam microscopy, Ultramicroscopy. 109 (2009) 1229-1235. https://doi.org/10.1016/J.ULTRAMIC.2009.05.008.

[46] K. Lepinay, F. Lorut, Three-dimensional semiconductor device investigation using focused ion beam and scanning electron microscopy imaging (fib/sem tomography), Microsc. Microanal. 19 (2013) 85-92. https://doi.org/10.1017/S1431927612014031.

[47] N. Hilairet, B. Reynard, Y. Wang, I. Daniel, S. Merkel, N. Nishiyama, S. Petitgirard, High-Pressure Creep of Serpentine, Interseismic Deformation, and Initiation of Subduction, Science (80-. ). 318 (2007) 1910-1913. https://doi.org/10.1126/science.1148494.

[48] L. Bezacier, B. Reynard, J.D. Bass, C. Sanchez-Valle, B. Van de Moortèle, Elasticity of antigorite, seismic detection of serpentinites, and anisotropy in subduction zones, Earth Planet. Sci. Lett. 289 (2010) 198-208. https://doi.org/10.1016/J.EPSL.2009.11.009.

[49] M. Schaffer, J. Wagner, Block lift-out sample preparation for 3D experiments in a dual beam focused ion beam microscope, Microchim. Acta. 161 (2008) 421-425. https://doi.org/10.1007/s00604-007-0853-5.

[50] P.R. Shearing, J. Golbert, R.J. Chater, N.P. Brandon, 3D reconstruction of SOFC anodes using a focused ion beam lift-out technique, Chem. Eng. Sci. 64 (2009) 3928-3933. https://doi.org/10.1016/j.ces.2009.05.038.

[51] J.A.W. Heymann, M. Hayles, I. Gestmann, L.A. Giannuzzi, B. Lich, S. Subramaniam, Site-specific 3D imaging of cells and tissues with a dual beam microscope, J. Struct. Biol. 155 (2006) 63-73. https://doi.org/10.1016/j.jsb.2006.03.006. 
[52] C. Villinger, H. Gregorius, C. Kranz, K. Höhn, C. Münzberg, G. Von Wichert, B. Mizaikoff, G. Wanner, P. Walther, FIB/SEM tomography with TEM-like resolution for 3D imaging of high-pressure frozen cells, Histochem. Cell Biol. 138 (2012) 549-556. https://doi.org/10.1007/s00418-012-1020-6.

[53] G. Knott, H. Marchman, D. Wall, B. Lich, Serial Section Scanning Electron Microscopy of Adult Brain Tissue Using Focused Ion Beam Milling, J. Neurosci. 28 (2008) 2959_ 2964. https://doi.org/10.1523/jneurosci.3189-07.2008.

[54] H.Z. Wu, S.G. Roberts, G. Möbus, B.J. Inkson, Subsurface damage analysis by TEM and 3D FIB crack mapping in alumina and alumina/5vol.\%SiC nanocomposites, Acta Mater. 51 (2003) 149-163. https://doi.org/10.1016/S1359-6454(02)00387-7.

[55] E. Keehan, L. Karlsson, H.K.D.H. Bhadeshia, M. Thuvander, Three-dimensional analysis of coalesced bainite using focused ion beam tomography, Mater. Charact. 59 (2008) 877882. https://doi.org/10.1016/j.matchar.2007.07.011.

[56] Boyde A., Quantitative photogrammetric analysis and qualitative stereoscopic analysis of SEM images, J. Microsc. 98 (1973) 452-471.

[57] G. Piazzesi, Photogrammetry with the scanning electron microscope, J. Phys. E. 6 (1973) 392-396. https://doi.org/10.1088/0022-3735/6/4/023.

[58] J.E. Castle, P.A. Zhdan, Characterization of surface topography by SEM and SFM: Problems and solutions, J. Phys. D. Appl. Phys. 30 (1997) 722-740. https://doi.org/10.1088/0022-3727/30/5/004.

[59] W. Drzazga, J. Paluszynski, W. Slowko, Three-dimensional characterization of microstructures in a SEM, Meas. Sci. Technol. 17 (2006) 28-31. https://doi.org/10.1088/0957-0233/17/1/006.

[60] F. Marinello, P. Bariani, E. Savio, A. Horsewell, L. De Chiffre, Critical factors in SEM 3D stereo microscopy, Meas. Sci. Technol. 19 (2008). https://doi.org/10.1088/09570233/19/6/065705.

[61] T. Zhu, M.A. Sutton, N. Li, J.J. Orteu, N. Cornille, X. Li, A.P. Reynolds, Quantitative Stereovision in a Scanning Electron Microscope, Exp. Mech. 51 (2011) 97-109. https://doi.org/10.1007/s11340-010-9378-7.

[62] M. Eulitz, G. Reiss, 3D reconstruction of SEM images by use of optical photogrammetry software, J. Struct. Biol. 191 (2015) 190-196. https://doi.org/10.1016/j.jsb.2015.06.010.

[63] L.C. Gontard, R. Schierholz, S. Yu, J. Cintas, R.E. Dunin-Borkowski, Photogrammetry of the three-dimensional shape and texture of a nanoscale particle using scanning electron microscopy and free software, Ultramicroscopy. 169 (2016) 80-88. https://doi.org/10.1016/j.ultramic.2016.07.006.

[64] V.N. Tondare, J.S. Villarrubia, A.E. Vladár, Three-Dimensional (3D) Nanometrology Based on Scanning Electron Microscope (SEM) Stereophotogrammetry, Microsc. Microanal. 23 (2017) 967-977. https://doi.org/10.1017/S1431927617012521. 
[65] A.V. Krishna, O. Flys, V. V. Reddy, B.G. Rosén, Surface topography characterization using 3D stereoscopic reconstruction of SEM images, Surf. Topogr. Metrol. Prop. 6 (2018). https://doi.org/10.1088/2051-672X/aabde1.

[66] Q. Shi, S. Roux, F. Latourte, F. Hild, D. Loisnard, N. Brynaert, Measuring topographies from conventional SEM acquisitions, Ultramicroscopy. 191 (2018) 18-33. https://doi.org/10.1016/j.ultramic.2018.04.006.

[67] R. Podor, X. Le Goff, T. Cordara, M. Odorico, J. Favrichon, L. Claparede, S. Szenknect, N. Dacheux, 3D-SEM height maps series to monitor materials corrosion and dissolution, Mater. Charact. 150 (2019) 220-228. https://doi.org/10.1016/J.MATCHAR.2019.02.017. 